\title{
Vortex Dynamics and Fractal Structures in Reactive and Nonreactive Richtmyer-Meshkov Instability
}

M. Bambauer, ${ }^{1}$ a) N. Chakraborty, ${ }^{2}$ M. Klein, ${ }^{1}$ and J. Hasslberger ${ }^{1}$

${ }^{1)}$ Bundeswehr University Munich, Department of Aerospace Engineering, 85577, Neubiberg, Germany

${ }^{2)}$ Newcastle University, School of Engineering, Newcastle upon Tyne, NE1 7RU, United Kingdom

(Dated: 23 March 2021)

Hydrodynamic instabilities caused by shock-flame interactions are a fundamental challenge in the accurate prediction of explosion loads in the context of nuclear and process plant safety. To investigate the Richtmyer-Meshkov instability (RMI), a series of three dimensional numerical simulations of shock-flame interactions is performed, including lean, stoichiometric and nonreactive homogeneous $\mathrm{H}_{2} / \mathrm{Air}$ mixtures. The equivalence ratio has a strong influence on the achievable flame wrinkling and mixing, by impacting key physical parameters such as the heat release parameter, flame thickness and reactivity. The reactivity is found to be a decisive factor in the evolution of the wrinkled flame brush, as it can cause burnout of the developing fresh gas cusps and wrinkled structures. The importance of reactivity is further emphasized by comparisons to a nonreactive case. Analysis of the enstrophy (energy equivalent of vorticity) transport terms shows, that baroclinic torque is dominant during shock-flame interactions. After the shock interaction, the vortex stretching, dissipation and dilatation terms gain in importance significantly. A power-law based modeling approach of the flame wrinkling is investigated by explicitly filtering the present simulation data. The values determined for the fractal dimension show a non-linear dependency on the chosen equivalence ratio, whereas the inner cut-off scale is found to be approximately independent of the equivalence ratio for the investigated cases.

a)Electronic mail: maximilian.bambauer@unibw.de 


\section{INTRODUCTION}

The interaction of a density gradient $\nabla \rho$ as present at the interface between a light and heavy fluid and a pressure gradient $\nabla p$ can lead to hydrodynamic instability, commonly referred to as the Richtmyer-Meshkov instability (RMI) ${ }^{1,2}$ or the Rayleigh-Taylor instability (RTI) ${ }^{3,4}$. In the case of RTI the pressure gradient is caused by constant (e.g. gravity) or time varying acceleration ${ }^{5}$, while the RMI is caused by the pressure gradient across a shock wave (impulsive acceleration). The misalignment of $\nabla p$ and $\nabla \rho$ leads to the production of vorticity, amplifying small disturbances across the interface and subsequently causing wrinkling and increased mixing of heavy and light fluid. An extensive review of occurrences for RMI and RTI in nature and technology, alongside common modeling approaches has been compiled by Zhou ${ }^{6,7}$ and Zhou et al. ${ }^{8}$. Notable use-cases in the non-combustive context are inertial confinement fusion ${ }^{9}$ and the modeling of supernovae explosions ${ }^{10}$. This work focuses on the RMI in the context of combustion, where it plays an important role as a mechanism for flame acceleration (FA) and deflagration-to-detonation transition (DDT) in geometrically confined explosions ${ }^{11,12}$. In this setting the RMI is caused by a shock wave $(\nabla p)$ interacting with a flame $(\nabla \rho)$, after being reflected from an obstacle or wall ${ }^{13,14}$. Figure 1 shows the mechanism, responsible for the amplification of disturbances on the flame surface after interacting with the shock-wave. Notable is also the phase-reversal effect in Fig. 1 (left), that occurs when the shock wave travels from high to low density. The influence of the RMI can be a decisive factor for DDT, as it causes heavy wrinkling of the flame brush region and subsequently a strong increase in the integral reaction rate ${ }^{15}$. In nuclear and process plant safety research, the accurate prediction of DDT and explosion loads poses a major challenge ${ }^{16}$. The heavy computational demands caused by the wide time- and length-scale spectra, associated with the simulation of the chemistry and turbulent flow in these cases ${ }^{17}$ only allow for the qualitative prediction of explosion loads in current commercial applications. By including small-scale RMI effects via a subgrid model, the accuracy of large eddy simulations (LES) or unsteady Reynolds-averaged Navier-Stokes simulations (URANS) could be improved ${ }^{18}$. This work focuses on the influence of the equivalence ratio on the development of the RMI by comparing shock-flame interactions of a lean and stoichiometric $\mathrm{H}_{2}$ /Air gas mixture. The equivalence ratio influences many important flame characteristics, such as flame speed, adiabatic flame temperature and flame thickness. In many reactor safety scenarios the gas mixture is typically assumed to be lean ${ }^{19}$, therefore analyzing and understanding the influence of these flame characteristics is an important step towards 
Vortex Dynamics and Fractal Structures in Reactive and Nonreactive RMI
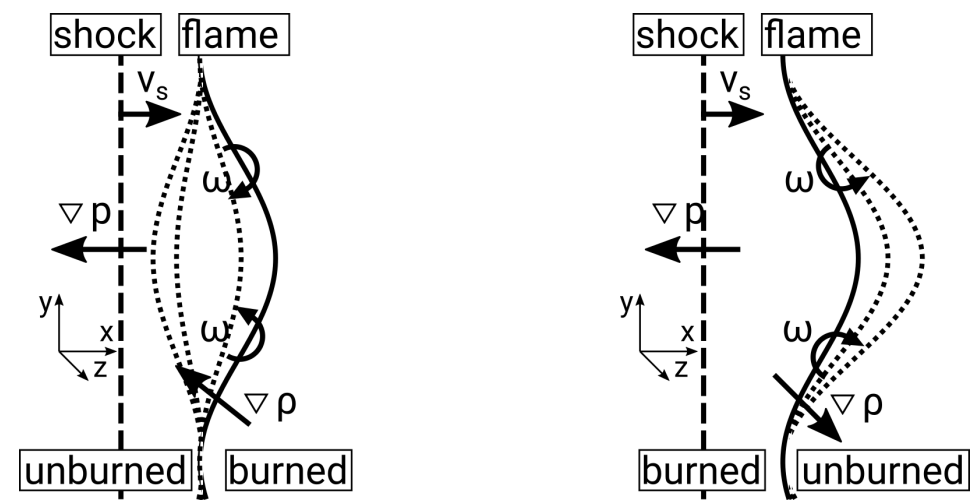

FIG. 1. Amplification of flame surface disturbance (-) and its temporal evolution (…*) due to baroclinic torque $(\omega)$ caused by the misalignment of $\nabla p$ and $\nabla \rho$. Left: shock wave (---) travels with velocity $V_{\mathrm{s}}$ from the unburned to the burned side, including phase reversal effect. Right: shock wave travels from the burned to the unburned side.

creating a subgrid model for this use case. An overview on the numerical methods used in the simulation is given in Sec. II, followed by an explanation of the case set-up in Sec. III. The simulation results are presented in Sec. IV, which includes a discussion on the temporal development of the flame surface area $A_{\mathrm{f}}$ and mixing width $\delta_{\mathrm{w}}$ (Sec. IV A) and a detailed analysis of individual terms in the enstrophy (energy-equivalent of the vorticity) transport equation in Sec. IV B. The discussion of the results is completed with Sec. IV C, investigating the fractal behavior of the wrinkled flame brush.

\section{NUMERICAL METHODS}

The high-fidelity 3D combustion simulation of the shock-flame interactions are carried out using the SENGA solver ${ }^{20}$. It solves for the compressible Navier-Stokes equations, including the total energy $e_{\mathrm{t}}$ (with the enthalpy $h$ ) and reaction progress variable $c$, given by Eqs. (1a) to (1d). 


$$
\begin{aligned}
\frac{\partial \rho}{\partial t}+\frac{\partial\left(\rho u_{k}\right)}{\partial x_{k}} & =0 \\
\frac{\partial\left(\rho u_{i}\right)}{\partial t}+\frac{\partial\left(\rho u_{k} u_{i}\right)}{\partial x_{k}}= & -\frac{\partial p}{\partial x_{i}}+\frac{\partial \tau_{k i}}{\partial x_{k}} \\
\frac{\partial\left(\rho e_{t}\right)}{\partial t}+\frac{\partial\left(\rho u_{k} e_{t}\right)}{\partial x_{k}}= & -\frac{\partial\left(p u_{k}\right)}{\partial x_{k}}+\frac{\partial\left(\tau_{k i} u_{i}\right)}{\partial x_{k}} \\
& +\frac{\partial}{\partial x_{k}}\left[\lambda \frac{\partial T}{\partial x_{k}}\right]-\frac{\partial}{\partial x_{k}}\left[h \rho D \frac{\partial c}{\partial x_{k}}\right] \\
\frac{\partial(\rho c)}{\partial t}+\frac{\partial\left(\rho u_{k} c\right)}{\partial x_{k}}= & \dot{\omega}+\frac{\partial}{\partial x_{k}}\left[\rho D \frac{\partial c}{\partial x_{k}}\right]
\end{aligned}
$$

The reaction progress variable $\mathrm{c}$ can be defined with the fuel mass fraction $Y_{\mathrm{F}}$ :

$$
c=\frac{Y_{\mathrm{F}}-Y_{\mathrm{F}}^{\mathrm{u}}}{Y_{\mathrm{F}}^{\mathrm{b}}-Y_{\mathrm{F}}^{\mathrm{u}}}
$$

where superscripts $\mathrm{u}$ and $\mathrm{b}$ denote unburnt and burnt gas quantities respectively. In fresh gases $c=0$ and in burnt gases $c=1$. Using the specific gas constant $R_{\mathrm{S}}$, the specific isochoric heat capacity $c_{\mathrm{v}}$ and the specific heat of reaction $H$ the equations of state are:

$$
\begin{aligned}
p & =\rho R_{\mathrm{s}} T \\
e_{\mathrm{t}} & =c_{\mathrm{v}}\left(T-T_{\mathrm{ref}}\right)+0.5 u_{k} u_{k}+H(1-c)
\end{aligned}
$$

The thermodynamic properties $\left(\lambda, c_{\mathrm{p}}, c_{\mathrm{V}}, \rho D\right)$ are approximated as constant (temperature independent). Usage of a detailed chemistry model would lead to large computational costs ${ }^{21,22}$ and is not justified for the study conducted here, since the RMI observed in this use-case is mainly dominated by the fluid dynamics of the shock-flame interaction. In the context of turbulent reactive flows, Arrhenius-type approaches are commonly found in literature ${ }^{23}$. The chemical source term ( $\dot{\omega}$ in Eq. (1d)) is expressed using an irreversible Arrhenius expression

$$
\dot{\omega}=\rho B(1-c) \exp \left[\frac{-E_{\mathrm{ac}}}{R T}\right],
$$

where $B, E_{\text {ac }}$ and $R$ denote the pre-exponential factor, the activation energy and the molar gas constant. The dimensionless temperature $T^{*}$ is defined as $T^{*}=\left(T-T_{0}\right) /\left(T_{\mathrm{ad}}-T_{0}\right)$, where $T_{\mathrm{ad}}$ is the adiabatic flame temperature and $T_{0}$ is the reference temperature corresponding to the initial state of the unburned $\mathrm{H}_{2}$ /Air gas-mixture. By defining the Zeldovich number as $\beta_{\mathrm{z}}=E_{\mathrm{ac}}\left(T_{\mathrm{ad}}-\right.$ $\left.T_{0}\right) /\left(R T_{\mathrm{ad}}^{2}\right)$ and the heat release parameters $\tau_{\mathrm{h}}=\left(T_{\mathrm{ad}}-T_{0}\right) / T_{0}$ and $\alpha_{\mathrm{h}}=\tau_{\mathrm{h}} /\left(1+\tau_{\mathrm{h}}\right)$, Eq. (4) can be written as: 


$$
\dot{\omega}=\rho B \exp \left[-\frac{\beta_{\mathrm{z}}}{\alpha_{\mathrm{h}}}\right](1-c) \exp \left[-\frac{\beta_{\mathrm{z}}\left(1-T^{*}\right)}{1-\alpha_{\mathrm{h}}\left(1-T^{*}\right)}\right]
$$

For the cases presented in this work a constant Zeldovich number of $\beta_{\mathrm{Z}}=5.0$ is assumed ${ }^{24}$ and the values chosen for $\tau_{\mathrm{h}}$ are listed in Tab. I.

The viscous stress tensor $\tau_{i j}$ is defined as:

$$
\tau_{i j}=\mu\left[\frac{\partial u_{i}}{\partial x_{j}}+\frac{\partial u_{j}}{\partial x_{i}}\right]-\frac{2}{3} \mu \delta_{i j}\left[\frac{\partial u_{k}}{\partial x_{k}}\right]
$$

Using reference values for density $\rho_{0}$, thermal conductivity $\lambda_{0}$, mass diffusivity $D_{0}$, specific heat capacity $c_{\mathrm{p}, 0}$ respective $c_{\mathrm{v}, 0}$ and dynamic viscosity $\mu_{0}$, additional dimensionless quantities such as the reference Reynolds number $\mathrm{Re}_{0}$, Prandtl number Pr and the Lewis number Le are defined as:

$$
\operatorname{Re}_{0}=\frac{\rho_{0} u_{0} l_{0}}{\mu_{0}} \quad \operatorname{Pr}=\frac{\mu_{0} c_{\mathrm{p}, 0}}{\lambda_{0}} \quad \text { Le }=\frac{\lambda_{0}}{\rho_{0} D_{0} c_{\mathrm{p}, 0}}
$$

The reference velocity $u_{0}$ is set to the laminar burning velocity $S_{\mathrm{L}}$. The reference length $l_{0}$ is defined as $\approx 250 \delta_{\mathrm{th}, \mathrm{st}}$, with $\delta_{\mathrm{th}, \mathrm{st}}$ being the initial thermal laminar flame thickness for the stoichiometric mixture. The reference speed of sound is defined as $a_{0}=\sqrt{\gamma R_{S} T_{0}}$, where $\gamma=c_{\mathrm{p}, 0} / c_{\mathrm{v}, 0}$ is the heat capacity ratio and $R_{\mathrm{S}}$ is the specific gas constant. With this, a reference Mach number $\mathrm{Ma}_{0}=u_{0} / a_{0}$ can be defined. Both $\mathrm{Ma}_{0}$ and $\mathrm{Re}_{0}$ are functions of $u_{0}=S_{\mathrm{L}}$ and therefore dependent on flame properties. The shock Mach number is defined as $M_{\mathrm{s}}=V_{\mathrm{s}} / a$, where $V_{\mathrm{s}}$ is the velocity of the shock wave and $a=\sqrt{\gamma R_{S} T}$ is the local speed of sound. Another possible definition of the Reynolds number involves the usage of the velocity jump $\Delta v$ induced by the shock on the flame surface. The difficulty in this definition lies in the fact that the velocity jump is dependent on the equivalence ratio and changes for shock and reshock. In addition, the velocity jump for the reshock can be difficult to determine analytically and is therefore not a suitable base for comparison. The reference Mach number $\mathrm{Ma}_{0}$ can be interpreted as a dimensionless flame speed, since $\mathrm{Ma}_{0}=S_{\mathrm{L}} / a_{0}$ and therefore a measure for the reactivity of the flame.

A low-storage 3rd order explicit Runge-Kutta scheme ${ }^{25}$ is utilized for temporal discretization. The spatial derivatives of all convective terms are calculated with the 5th order WENO-5 method by Jiang and Shu ${ }^{26}$, using the scalar Lax-Friedrichs flux splitting procedure described by Shu ${ }^{27}$. The scheme provides minimal-dissipation shock capturing capabilities without numerical oscillations for the investigated cases. In order to assess the shock capturing capabilities of the scheme 
Vortex Dynamics and Fractal Structures in Reactive and Nonreactive RMI
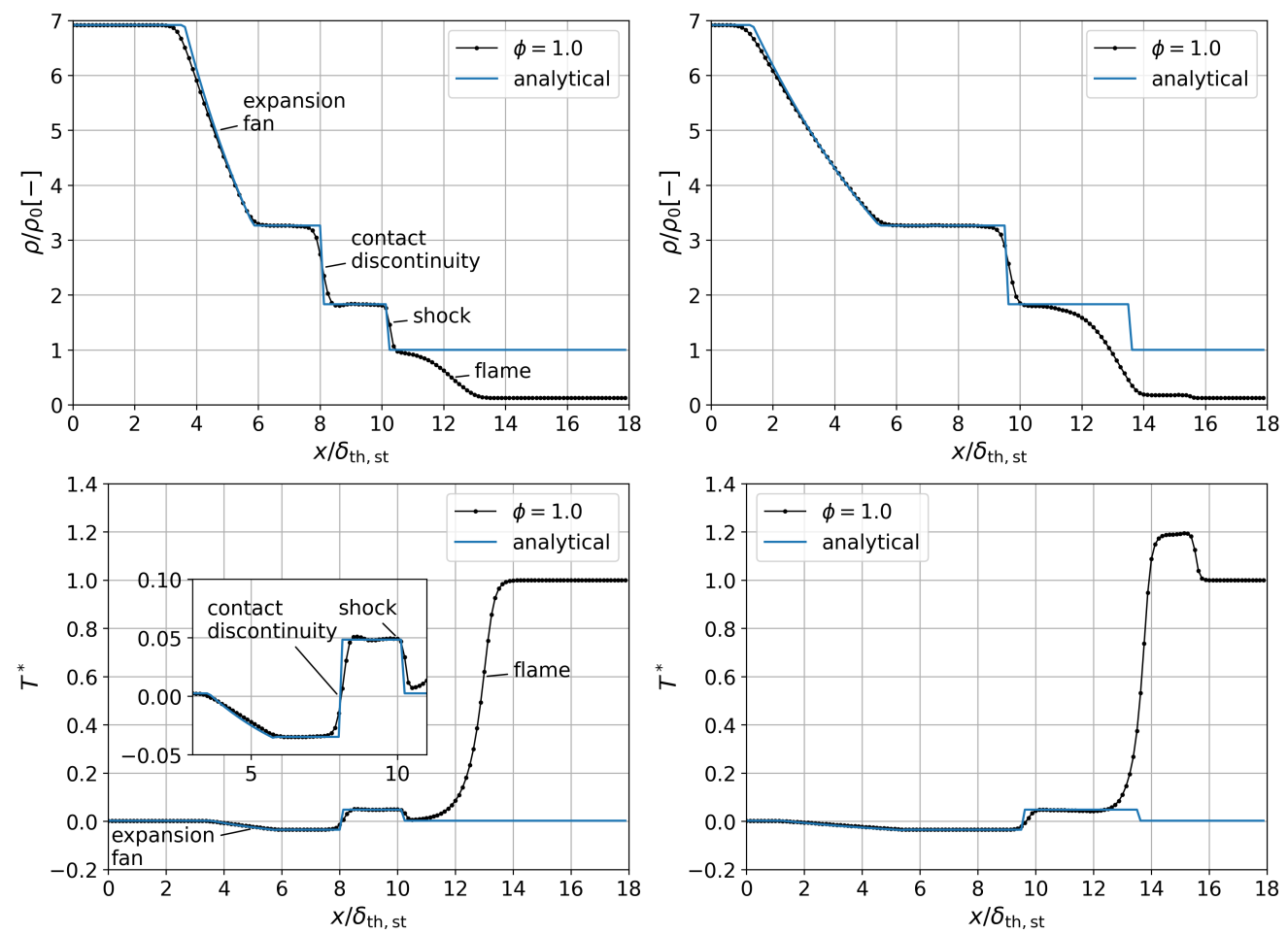

FIG. 2. Comparison of the numerical and analytical reactive shock-tube solution for $\phi=1.0$ and $\mathrm{Ma}_{\mathrm{s}}=1.5$. Normalized density (top) and normalized temperature (bottom) with $T^{*}=\left(T-T_{0}\right) /\left(T_{\mathrm{ad}}-T_{0}\right)$ before (left column) and after (right column) shock-flame interaction.

in a reactive case, the $1 \mathrm{D}$ simulation results of a $\mathrm{Ma}_{\mathrm{s}}=1.5$ shock wave interacting with a $\phi=1.0$ flame (Table I) is shown in Fig. 2. At the beginning of the simulation the shock is being initialized as a sharp discontinuity and then slightly smoothened by the WENO-5 scheme, which can resolve the shock within 2-3 nodes. The left column of Fig. 2 shows the normalized density and normalized temperature profiles before the shock interacts with the flame. Note that the normalized temperature is defined as $T^{*}=\left(T-T_{0}\right) /\left(T_{\mathrm{ad}}-T_{0}\right)$ and is $T^{*}=0$ in the unshocked and unburned part of the gas $\left(T=T_{0}\right)$ and $T^{*}=1$ in the unshocked and burned part of the gas $\left(T=T_{\mathrm{ad}}\right)$. In the right column of Fig. 2 the shock has passed the flame. The shock remains resolved sharply before and after the flame interaction and no oscillations are visible. Note that the analytical solution does not take the flame into account, which causes an offset in the shock position between numerical and analytical solution after the flame has been passed. 


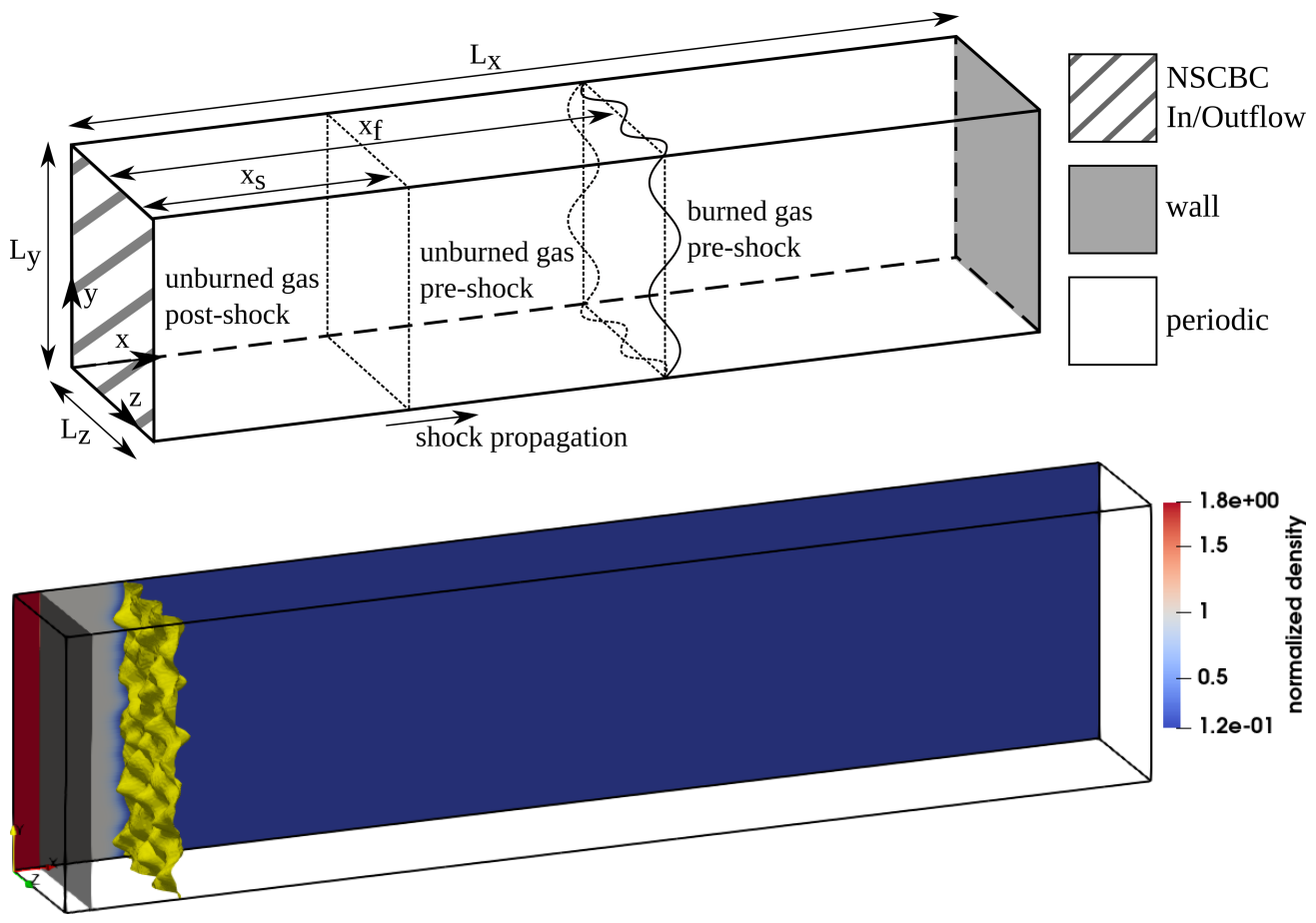

FIG. 3. Top: Setup schematic and boundary conditions with shock propagating from left to right and statistically planar flame. Bottom: 3D view and normalized density slice of initial case setup for $\phi=$ 1.0. Flame and shock wave are represented by iso-surfaces of $c=0.5$ (yellow) and $\rho / \rho_{0}=1.4$ (grey), respectively.

\section{SIMULATION SETUP}

Figure 3 shows a schematic of the simulation domain, consisting of a rectangular channel divided into areas of unburned and burned gas by a statistically planar flame. At $x=0$ a modified Navier-Stokes characteristic boundary condition (NSCBC) is implemented, allowing for both outflow and inflow of fluid ${ }^{28}$. An adiabatic wall boundary condition is applied at $x=L_{\mathrm{x}}$, to enable the reflection of incoming shock waves. The boundary conditions in y and z-direction are periodic. At non-periodic boundaries a switch to an 8th-order central scheme is made. The stencil is then reduced stepwise down to second order and finally to a one-sided 2nd order scheme, as the boundary is approached.

An algorithm proposed by Tritschler et al. ${ }^{29}$ is implemented to produce a well defined initial disturbance of the flame surface. All presented cases are initialized with the flame disturbance field shown in Fig. 4, where the color represents the displacement in x-direction of the statistically pla- 
Vortex Dynamics and Fractal Structures in Reactive and Nonreactive RMI
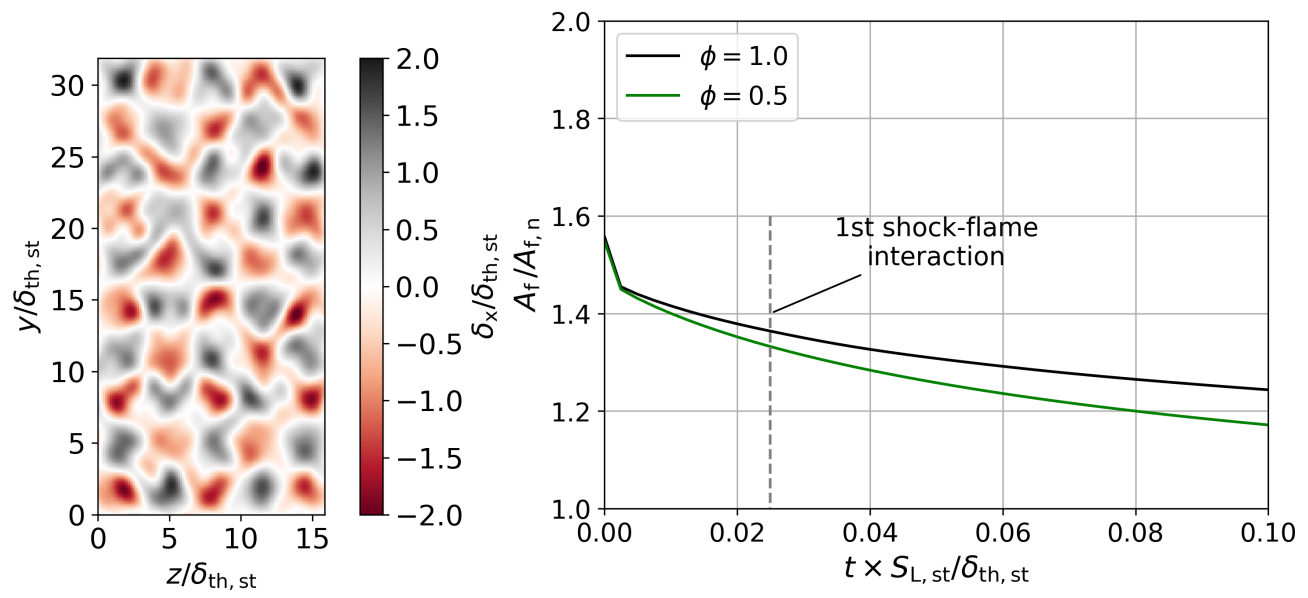

FIG. 4. Left: Normalized distortion $\delta_{\mathrm{x}} / \delta_{\mathrm{th}, \mathrm{st}}$ of the statistically planar flame front. Right: Development of the disturbed flame surface in a hypothetical case without shock interaction for $\phi=1.0$ and $\phi=0.5$.

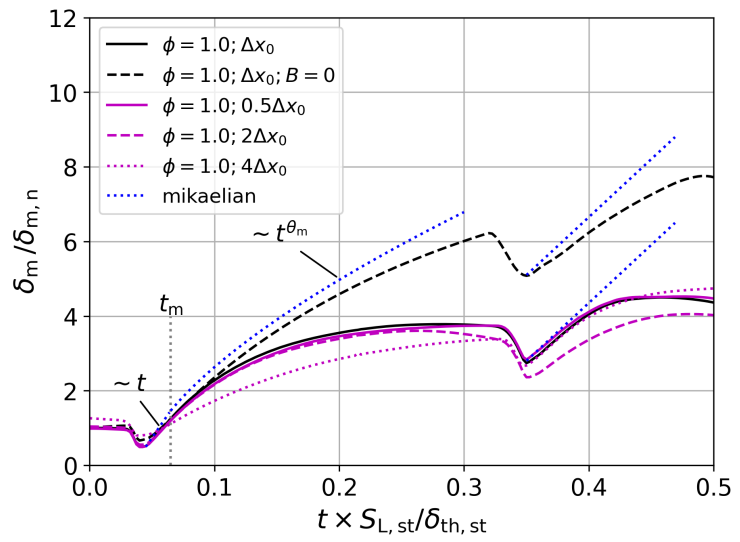

FIG. 5. Normalized mixing width over time for $\phi=1.0$ and nonreactive gas mixture $(B=0)$ at different grid resolutions. Comparison with model predictions of the normalized mixing width.

nar flame surface. The displacement values are derived from a base oscillation with a wavenumber of $10 \pi / L_{\mathrm{y}}$ and superimposed with multi-wavenumber oscillations of small amplitudes to enable a quasi-stochastic disturbance of the flame front. As visible in Fig. 4 (left) the wavelength of the base oscillation is much larger than $\delta_{\mathrm{th}, \mathrm{st}}$ and therefore well resolved. The perturbations will not change significantly before the first shock interaction, as the time until shock interaction is very small $\left(t \times S_{\mathrm{L}, \mathrm{st}} / \delta_{\mathrm{th}, \mathrm{st}}=0.025 \ll 1\right)$. Fig. 4 (right) shows the replanarization of the distorted flame surface in a hypothetical case without shock interaction. A sharp decline is visible at the beginning of the simulation as the smallest perturbations are smoothed due to numerical diffusion. At later times the flame surface decreases slightly due to physical diffusion. 
The grid sensitivity of the instability growth is shown alongside predictions of the Mikaelian model $^{30}$ in Fig. 5. In a nonreactive context this model can be used to assess the growth of the mixing layer width. The model is applied to the $\phi=1.0$ and the nonreactive case, where $\delta_{\mathrm{m}}$ scales linearly $\left(\delta_{\mathrm{m}} \sim t\right)$ immediately after the shock interaction before transitioning at $t=t_{\mathrm{m}}$ to non-linear behaviour $\left(\delta_{\mathrm{m}} \sim t^{\theta_{\mathrm{m}}}\right)$. According to the model, the mixing layer width $\delta_{\mathrm{m}}$ in the linear regime and nonlinear regime can be approximated as:

$$
\begin{aligned}
\delta_{\mathrm{m}, \operatorname{lin}}(t) & =\delta_{\mathrm{m}, 0}+2 \alpha_{\mathrm{m}} A_{\mathrm{atw}} \Delta v t \quad \text { for } \quad t \leq t_{\mathrm{m}} \\
\delta_{\mathrm{m}, \theta}(t) & =\delta_{\mathrm{m}, \operatorname{lin}}\left(t=t_{\mathrm{m}}\right)\left(1+\frac{2 \alpha_{\mathrm{m}} A_{\mathrm{atw}} \Delta v}{\delta_{\mathrm{m}, \operatorname{lin}}\left(t=t_{\mathrm{m}}\right) \theta_{\mathrm{m}}}\left(t-t_{\mathrm{m}}\right)\right)^{\theta_{\mathrm{m}}} \quad \text { for } \quad t \geq t_{\mathrm{m}}
\end{aligned}
$$

The transition time is approximated using $t_{\mathrm{m}} \approx \delta_{\mathrm{m}, 0} \beta_{\mathrm{m}} / \Delta v$, where $\delta_{\mathrm{m}, 0}$ is the mixing width at the start of the shock-flame interaction, $\Delta v$ is the velocity jump at the mixing interface caused by the shock and $\beta_{\mathrm{m}}=6$ is a nondimensional constant. For the first shock interaction $\Delta v$ equals the post shock velocity $u_{\mathrm{s}}=124 S_{\mathrm{L}}$, which can be calculated using the following nondimensional shock relation:

$$
\frac{u_{\mathrm{s}}}{S_{\mathrm{L}}}=\frac{1+\frac{\gamma-1}{\gamma+1}\left(\mathrm{Ma}_{\mathrm{s}}^{2}-1\right)}{\mathrm{Ma}_{\mathrm{s}}^{2}}\left(1-\frac{\mathrm{Ma}_{\mathrm{s}}}{\mathrm{Ma}_{0}}\right)+\frac{\mathrm{Ma}_{\mathrm{s}}}{\mathrm{Ma}_{0}}
$$

Eq. (9) is derived from the normal shock relations ${ }^{31}$ using $u_{0}=S_{\mathrm{L}}$ as the pre shock velocity, $T_{0}$ as the pre shock temperature and $V_{\mathrm{s}} / S_{\mathrm{L}}=\mathrm{Ma}_{\mathrm{s}} / \mathrm{Ma}_{0}$. The growth parameters $\alpha_{\mathrm{m}}$ and $\theta_{\mathrm{m}}$ are approximated to $\alpha_{\mathrm{m}} \approx 0.139$ and $\theta_{\mathrm{m}} \approx 0.636$, using the following equations from Dimonte and Schneider ${ }^{32}$

$$
\begin{gathered}
\alpha_{\mathrm{m}} \approx 0.07\left(1+\left[\left(1+A_{\mathrm{atw}}\right) /\left(1-A_{\mathrm{atw}}\right)\right]^{0.33}\right), \\
\theta_{\mathrm{m}} \approx 0.25\left(1+\left[\left(1+A_{\mathrm{atw}}\right) /\left(1-A_{\mathrm{atw}}\right)\right]^{0.21}\right),
\end{gathered}
$$

where the Atwood number $A_{\mathrm{atw}}=\left(\rho_{\mathrm{ub}}-\rho_{\mathrm{b}}\right) /\left(\rho_{\mathrm{ub}}+\rho_{\mathrm{b}}\right)=0.78$ describes a ratio of the burned $\left(\rho_{\mathrm{b}}\right)$ and unburned $\left(\rho_{\mathrm{ub}}\right)$ gas density. In literature ${ }^{33,34}$, typical values for the growth parameter $\theta_{\mathrm{m}}$ have been shown to be between 0.213 and 0.666 . Using the values above, the growth rate in the linear regime $\dot{\delta}_{\mathrm{m} \text {,lin }}=2 \alpha_{\mathrm{m}} A_{\mathrm{atw}} \Delta v$ can be calculated to $\dot{\delta}_{\mathrm{m}, \text { lin }}=26.9 S_{\mathrm{L}}$. A perfect fit of the model and the simulation results is not expected, since the transition time $t_{\mathrm{m}}$ and Eqs. (10a) to (10b) are approximations (see Mikaelian ${ }^{30}$ for details). Nevertheless, the comparison emphasizes the similarities and differences between the reactive and the nonreactive case in terms of mixing width growth. Directly after the shock flame interaction the mixing width scales linearly for both 
Vortex Dynamics and Fractal Structures in Reactive and Nonreactive RMI
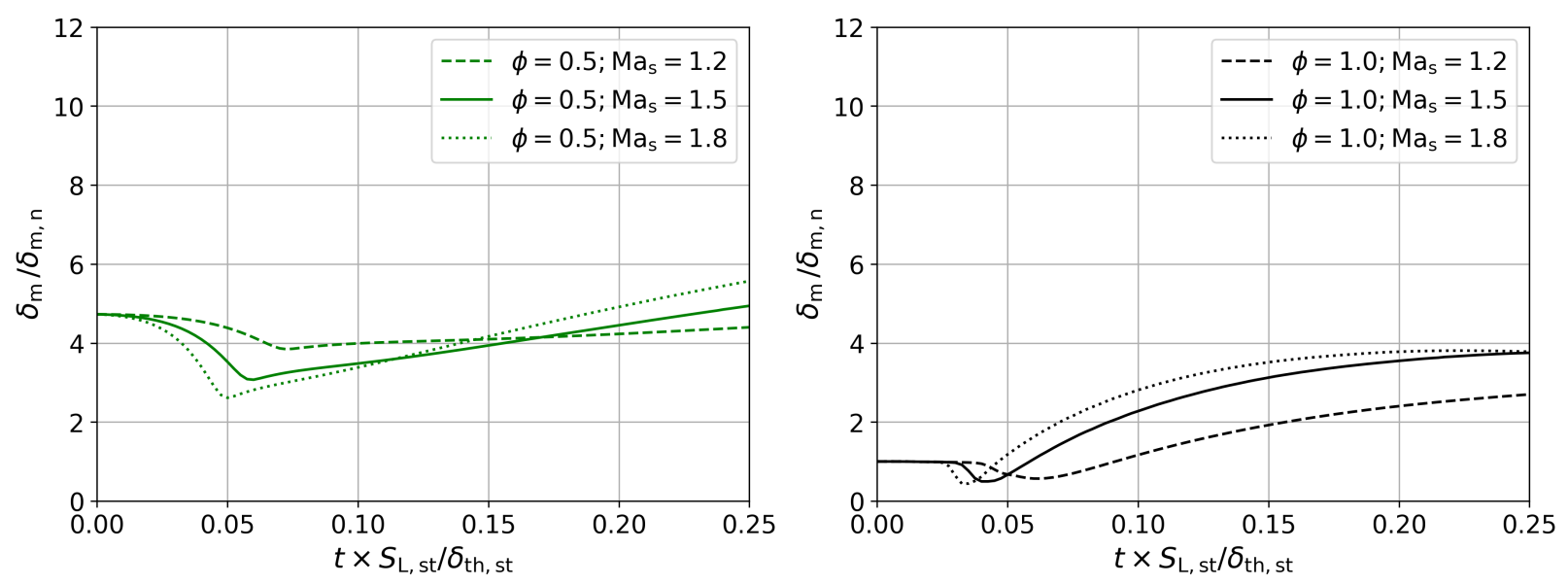

FIG. 6. Normalized mixing width over time for $\phi=0.5$ (left) and $\phi=1.0$ (right). Comparison of a shockflame interaction at Mach numbers 1.2, 1.5 and 1.8.

cases. After transitioning to the power law the differences between both cases become apparent, as the mixing width growth diminishes quickly for the reactive case. Decreasing the grid resolution (cases $2 \Delta x_{0}$ and $4 \Delta x_{0}$ ) causes an underestimation of the mixing width, since small perturbations can no longer be resolved. The fine $\left(0.5 \Delta x_{0}\right)$ and the base grid $\left(\Delta x_{0}\right)$ follow the linear trend of the model by Mikaelian ("mikaelian" in Fig. 5) ${ }^{30}$ well and are in close agreement with each other during the nonlinear portion of the shock flame interaction. Hence, the results analyzed in the following can be considered as grid-convergent.

Equation (8a) also provides an explanation on the effect of shock Mach number variations as shown in Fig. 6. Increasing $\mathrm{M}_{\mathrm{s}}$ leads to an increase of the velocity jump $\Delta v$ which directly increases the initial growth rate of the mixing width. Comparing the mixing width growth between $\phi=0.5$ and $\phi=1.0$ reveals a much stronger non-linear behavior for $\phi=1.0$, whereas the growth is mostly linear for $\phi=0.5$. This is caused by the increased reactivity in the stoichiometric case and will be further discussed in Sec. IV, where a detailed analysis of the effects of shock and reshock on the flame brush will be given.

The simulation parameters of the cases investigated in this work are listed in Tab. I. The two main cases are based on a $\mathrm{H}_{2} /$ Air gas mixture at an equivalence ratio of $\phi=0.5$ (lean case) and $\phi=1$ (stoichiometric case). Based on the setup of $\phi=0.5$, the parameter variations $\mathrm{A}, \mathrm{B}, \mathrm{C}$ and $\mathrm{D}$ are performed, where Le, $\mathrm{Re}_{0}, \tau_{\mathrm{h}}$ and $\mathrm{Ma}_{0}$ are successively changed to the parameters corresponding to the $\phi=1$ case. Additionally a nonreactive variation of the stoichiometric case is presented, where the pre-exponential factor is set to $B=0$, hence deactivating the chemical 
source term $\dot{\omega}=0$ (Eq. (5)). For all cases, the shock Mach number is set to $\mathrm{Ma}_{\mathrm{s}}=1.5$. The set-up values for $\phi=0.5$ and $\phi=1$ are calculated using the Cantera software ${ }^{35}$ and the GRI-MECH 3.0 database at $T_{0}=298.15 \mathrm{~K}$ and $p_{0}=1$ bar. The laminar flame speed $S_{\mathrm{L}}$ is calculated from Cantera to be $2.27 \mathrm{~m} / \mathrm{s}$ for $\phi=1.0$ and $0.385 \mathrm{~m} / \mathrm{s}$ for $\phi=0.5$. The pre-exponential factor $B$ is calculated within a separate pre-processing step in SENGA, using the input parameters from Tab. I, so that the corresponding $S_{\mathrm{L}}$ is achieved. The simplest approach when calculating the Lewis number (Eq. (7)) for a gas mixture is to use the mass diffusion coefficient of the deficient reactant. For a lean mixture $(\phi<1) \mathrm{H}_{2}$ is the deficient reactant and for a fuel rich mixture $(\phi>1) \mathrm{O}_{2}$ is the deficient reactant. In the limit of $(\phi \ll 1)$ the $\mathrm{H}_{2}$-Lewis number approaches $\mathrm{Le}_{\mathrm{H}_{2}} \approx 0.3$ and for $(\phi \gg 1)$ the $\mathrm{O}_{2}$-Lewis number approaches $\mathrm{Le}_{\mathrm{O}_{2}} \approx 2.1$. To avoid a jump at $\phi=1$, an effective (also known as reduced) Lewis number Le $e_{\text {eff }}$ is defined, that provides a smooth transition between $\mathrm{Le}_{\mathrm{H}_{2}}$ and $\mathrm{Le}_{\mathrm{O}_{2}}$ over the whole range of $\phi$. The following model for calculating $\mathrm{Le}_{\mathrm{eff}}$ is taken from Bechtold and Matalon ${ }^{36}$, with $\mathrm{Le}_{\mathrm{H}_{2}}$ and $\mathrm{Le}_{\mathrm{O}_{2}}$ being provided by Cantera.

$$
\mathrm{Le}_{\mathrm{eff}}=1+\frac{\left(\mathrm{Le}_{\mathrm{H}_{2}}-1\right)+\left(\mathrm{Le}_{\mathrm{O}_{2}}-1\right) A_{\mathrm{Le}}}{1+A_{\mathrm{Le}}}
$$

The factor $A_{\mathrm{Le}}$ is defined by $A_{\mathrm{Le}}=1+\beta_{\mathrm{z}}(\tilde{\phi}-1)$ with $\tilde{\phi}=\max (1 / \phi, \phi)$. For the sake of simplicity the subsequent discussion (including Tab. I) will refer to the effective Lewis number only as Lewis number or Le. Another approach for calculating an effective diffusivity, suitable for turbulent mixing of reactive species, is presented by O'Brien ${ }^{37}$.

In Sec. IV the evolutions of the normalized flame surface area $A_{\mathrm{f}} / A_{\mathrm{f}, \mathrm{n}}$ and the normalized mixing width $\delta_{\mathrm{m}} / \delta_{\mathrm{m}, \mathrm{n}}$ are examined. As normalization constants $A_{\mathrm{f}, \mathrm{n}}$ and $\delta_{\mathrm{m}, \mathrm{n}}$, the cross section of the shock tube $L_{\mathrm{y}} \times L_{\mathrm{z}}$ and the initial mixing width for $\phi=1.0$ at $t=0$ are chosen. Eq. (12) shows the equations used to calculate $A_{\mathrm{f}}{ }^{38}$ and $\delta_{\mathrm{m}}{ }^{39}$ from the simulation data, where $\langle\cdot\rangle$ indicates averaging in the yz-plane. The definition of $A_{\mathrm{f}}$, which represents the total flame surface area evaluated over the entire simulation domain, is based on the volume integral of the generalized flame surface density (FSD) ${ }^{40}$.

$$
\begin{aligned}
A_{\mathrm{f}} & =\iiint_{V}|\nabla c| d V \\
\langle c\rangle & =\frac{1}{L_{\mathrm{y}} L_{\mathrm{z}}} \iint c d y d z \\
\delta_{\mathrm{m}} & =\int_{0}^{L_{\mathrm{x}}} 4\langle c\rangle(1-\langle c\rangle) d x
\end{aligned}
$$


Vortex Dynamics and Fractal Structures in Reactive and Nonreactive RMI

TABLE I. Input parameters for the simulation cases. The bold numbers indicate the successive changes from $\phi=0.5$ to the parameters corresponding to $\phi=1.0$.

\begin{tabular}{ccccccccc}
\hline \hline case & $\mathrm{Le}$ & $\mathrm{Re}_{0}$ & $\tau_{\mathrm{h}}$ & $\mathrm{Ma}_{0}$ & $A_{\mathrm{atw}}$ & $\mathrm{Ma}_{\mathrm{s}}$ & $\mathrm{Pr}$ & $\dot{\omega}$ \\
\hline$\phi=1.0$ & 1.1 & 1093 & 7.1 & 0.005609 & 0.780 & 1.5 & 0.47 & Eq. (5) \\
$\phi=0.875$ & 0.9 & 933 & 6.7 & 0.004721 & 0.766 & 1.5 & 0.475 & Eq. (5) \\
$\phi=0.75$ & 0.7 & 718 & 6.1 & 0.003576 & 0.752 & 1.5 & 0.49 & Eq. (5) \\
$\phi=0.625$ & 0.6 & 457 & 5.3 & 0.002241 & 0.726 & 1.5 & 0.50 & Eq. (5) \\
$\phi=0.5$ & 0.5 & 213 & 4.5 & 0.001025 & 0.691 & 1.5 & 0.52 & Eq. (5) \\
nonreactive & 1.1 & 1093 & 7.1 & 0.005609 & 0.780 & 1.5 & 0.47 & 0 \\
$\mathrm{~A}$ & $\mathbf{1 . 1}$ & 213 & 4.5 & 0.001025 & 0.691 & 1.5 & 0.52 & Eq. (5) \\
$\mathrm{B}$ & $\mathbf{1 . 1}$ & $\mathbf{1 0 9 3}$ & 4.5 & 0.001025 & 0.691 & 1.5 & 0.52 & Eq. (5) \\
$\mathrm{C}$ & $\mathbf{1 . 1}$ & $\mathbf{1 0 9 3}$ & $\mathbf{7 . 1}$ & 0.001025 & 0.780 & 1.5 & 0.52 & Eq. (5) \\
$\mathrm{D}$ & $\mathbf{1 . 1}$ & $\mathbf{1 0 9 3}$ & $\mathbf{7 . 1}$ & $\mathbf{0 . 0 0 5 6 0 9}$ & 0.780 & 1.5 & 0.52 & Eq. (5) \\
\hline \hline
\end{tabular}

In the context of LES sub-grid model closure, evaluating $A_{\mathrm{f}} / A_{\mathrm{f}, \mathrm{n}}$ and $\delta_{\mathrm{m}} / \delta_{\mathrm{m}, \mathrm{n}}$ can be of pivotal importance, since they can be related to the closure of the reaction rate term and the convective term.

All length dimensions are normalized by the thermal laminar flame thickness $\delta_{\text {th,st }}$ at $\phi=1$. The dimensions of the domain are $L_{\mathrm{x}} \times L_{\mathrm{y}} \times L_{\mathrm{z}}=128 \delta_{\mathrm{th}, \mathrm{st}} \times 32 \delta_{\mathrm{th}, \mathrm{st}} \times 16 \delta_{\mathrm{th}, \mathrm{st}}$ uniformly discretized by $1024 \times 256 \times 128$ grid points. The shock and flame are initialized at $x_{\mathrm{s}}=3.125 \delta_{\mathrm{th}, \mathrm{st}}$ and $x_{\mathrm{f}}=$ $12.5 \delta_{\mathrm{th}, \mathrm{st}}$ respectively. The initial thermal laminar flame thickness of the lean case is $\delta_{\mathrm{th}, \mathrm{le}} \approx 8 \delta_{\mathrm{th}, \mathrm{st}}$ with $\delta_{\text {th }}=1 / \max \left|\nabla T^{*}\right|$.

\section{RESULTS}

Figure 7 shows density slices in the xy-plane at different times for $\phi=1.0$ and $\phi=0.5$. The density is normalized using the unburned and unshocked reference density $\rho_{0}$. The shock is visible as a discontinuity in $\rho$, increasing $\rho / \rho_{0}$ on each passage through the shock tube. The simulation ends at $t \times S_{\mathrm{L}, \mathrm{st}} / \delta_{\mathrm{th}, \mathrm{st}}=1$. The time steps $t_{\mathrm{a}}, t_{\mathrm{b}}, t_{\mathrm{c}}, t_{\mathrm{d}} \times S_{\mathrm{L}, \mathrm{st}} / \delta_{\mathrm{th}, \mathrm{st}}=0.2,0.4,0.6,0.8$ show different stages of the RMI development. Sharp ${ }^{41}$ describes four different stages of development for the 
Vortex Dynamics and Fractal Structures in Reactive and Nonreactive RMI

phenomenologically similar RTI:

- initial linear growth of flame perturbations

- formation of fresh gas cusps

- development of wrinkled structures

- break up of distinct cusps and creation of a chaotic mixing layer

At $t_{\mathrm{a}}$ the shock wave, which is propagating from left to right, has already interacted with the flame surface for the first time, causing the creation of distinct fresh gas cusps, which extend into the burned gas mixture. The influence of the initial displacement (Fig. 4) can still be identified as the flame cusps are inverted and further disturbed by the RMI (Fig. 1). In the context of premixed flames interacting with pressure gradients, a similar cusp-creation effect has been observed by Lipatnikov et al. ${ }^{42}$ and Poludnenko ${ }^{43}$. The increased reactivity at $\phi=1.0$ causes a transverse burnout of the fresh gas cusps, leading to the creation of regions of strong negative curvature (sharp edges). At $t_{\mathrm{b}}$ the shock has been reflected from the back wall $\left(x=L_{\mathrm{x}}\right)$ and has interacted with the flame surface a second time (reshock). While the maxima of the initial disturbance can still be identified at this stage of the RMI, the distortions are increased further and small scale wrinkled structures emerge on the flame surface. The shock wave is partially reflected from the flame surface at $t_{\mathrm{b}}$. This partial reflection interacts with the flame shortly before $t_{\mathrm{c}}$, causing further wrinkling of the flame brush and the creation of a mixing region of burned and unburned gas. At this stage the influence of the equivalence ratio $\phi$ becomes especially apparent as locally emerging small scale wrinkles are burned out for $\phi=1.0$, leaving only larger scale structures with regions of high negative flame curvature in between. The reduction of perturbation growth due to diffusion is an important mechanism of reactive RMI and is discussed and evaluated by Attal and Ramaprabhu ${ }^{44}$. An effect of similar nature (decreasing the wrinkling), caused by molecular diffusion on a reactive flame surface is discussed by $\mathrm{Yu}$ and Lipatnikov ${ }^{45}$. The last slice at $t_{\mathrm{d}}$ shows the development of the RMI towards the end of the simulations. The overall structure of the flame and the differences between $\phi=1.0$ and $\phi=0.5$ do not change significantly to the state discussed at $t_{\mathrm{c}}$. At this late stage the flame surface area reduces a little, due to diffusion effects and the interaction and merging of neighboring wrinkled structures. In the burned gas region $\left(T=T_{\mathrm{ad}}\right)$ the velocity $V_{\mathrm{s}}$ of a shock wave travelling at Mach number $\mathrm{Ma}_{\mathrm{s}}$ is defined by $V_{\mathrm{s}}=\mathrm{Ma}_{\mathrm{s}} \sqrt{\gamma R_{\mathrm{s}} T_{\mathrm{ad}}}$. Since $T_{\mathrm{ad}}$ is higher for $\phi=1.0$ than for $\phi=0.5$, the shock waves propagate at different velocities inside the burned 
Vortex Dynamics and Fractal Structures in Reactive and Nonreactive RMI

gas mixture, creating a visible offset in the shock position between both cases. The development of the 3D flame brush is shown in Fig. 8. The flame surface is represented by a yellow iso-surface at $c=0.5$. This visualization further highlights the influence of the equivalence ratio on the flame brush development, which becomes particularly apparent after the reshock. From $t=t_{\mathrm{b}}$ to $t=t_{\mathrm{d}}$ large flame cusps are starting to develop and grow for $\phi=1.0$, whereas for $\phi=0.5$ the developing wrinkled structures are found to be of a much smaller scale.

\section{A. Flame Surface Area and Mixing Width Analysis}

In order to further assess the temporal development of the RMI, the evolution of the normalized flame surface area $A_{\mathrm{f}} / A_{\mathrm{f}, \mathrm{n}}$ and the normalized mixing width $\delta_{\mathrm{m}} / \delta_{\mathrm{m}, \mathrm{n}}$ is analyzed in Fig. 9. An additional nonreactive ( $\dot{\omega}=0)$ variation of $\phi=1.0$ is analyzed by setting $B=0$ in Eq. (5). Generally, two different phases in the evolution of $A_{\mathrm{f}} / A_{\mathrm{f}, \mathrm{n}}$ can be distinguished. The first phase is characterized by the initial growth during and shortly after interaction with the shock wave, caused by the production of baroclinic torque on the flame surface (see Sec. IV B for a more detailed discussion on the influence of baroclinic torque and enstrophy). The amount of baroclinic torque produced, is dependent on the magnitude of the pressure gradient $\nabla p$ across the shock, the density gradient $\nabla \rho$ across the flame and the misalignment of shock wave and flame surface. Since cases $\phi=1.0$ and $\phi=0.5$ are set up using the same initial flame disturbance and initial shock Mach number $\left(\mathrm{Ma}_{\mathrm{s}}=1.5\right)$, the only influencing factor left to explain the differences in the initial development of $A_{\mathrm{f}} / A_{\mathrm{f}, \mathrm{n}}$ is $\nabla \rho$. Increasing the equivalence ratio from $\phi=0.5$ to $\phi=1.0$ increases $\nabla \rho$ by significantly reducing the flame thickness and increasing $T_{\mathrm{ad}}$ and therefore increasing the density difference between the burned and unburned side of the flame. The flame thickness $\delta_{\mathrm{f}}$ (Fig. 9) is defined by $\delta_{\mathrm{f}}=V_{\mathrm{f}} / A_{\mathrm{f}}$, where the flame volume $V_{\mathrm{f}}$ is calculated by summation of all grid points with $0.01 \leq c \leq 0.99$. The increase in $\nabla \rho$ causes a higher baroclinic torque production and subsequently a steeper initial increase and higher maximum value in $A_{\mathrm{f}} / A_{\mathrm{f}, \mathrm{n}}$ for $\phi=1.0$ compared with $\phi=0.5$. At reshock the development of $A_{\mathrm{f}} / A_{\mathrm{f}, \mathrm{n}}$ shows a different behavior. The rise of $A_{\mathrm{f}} / A_{\mathrm{f}, \mathrm{n}}$ after the reshock is similar in both cases (and the nonreactive case) until $\phi=1.0$ reaches a peak and decreases rapidly, while $\phi=0.5$ continues to rise, surpassing the stoichiometric case. The differences to the first shock-flame interaction can be attributed to two reasons. First, at the reshock the flame thickness of $\phi=0.5$ is reduced significantly (Fig. 9), as the shock wave increases the pressure and compresses the flame, increasing $\nabla \rho$ for this case. Second, as visible 
Vortex Dynamics and Fractal Structures in Reactive and Nonreactive RMI
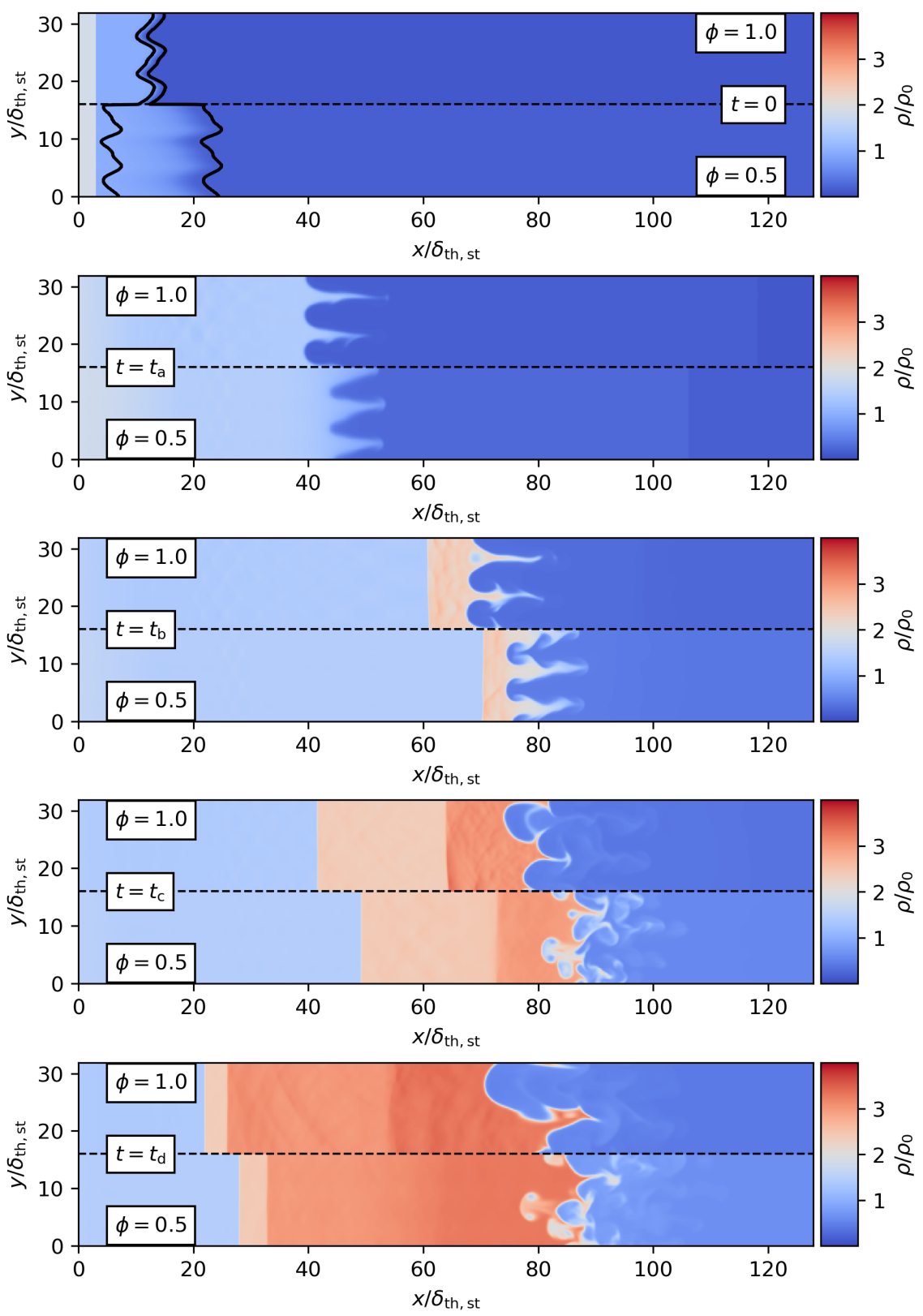

FIG. 7. Density slices $\left(z=L_{z} / 2\right)$ of the shock-flame interaction for $\phi=1.0$ and $\phi=0.5$. Timesteps $t_{\mathrm{a}}, t_{\mathrm{b}}, t_{\mathrm{c}}, t_{\mathrm{d}} \times S_{\mathrm{L}, \mathrm{st}} / \delta_{\mathrm{th}, \mathrm{st}}=0.2,0.4,0.6,0.8$. Iso-contours of the reaction progress variable $(c=0.02$ and $c=$ $0.98)$ show the difference in flame thickness at $t=0$.

in Fig. 7 at $t_{\mathrm{b}}$, the development of wrinkled structures on the flame surface are a big contributing factor on the overall growth of the flame surface area. As already mentioned in the discussion of Fig. 7 the increased reaction rate at $\phi=1.0$ locally burns out the wrinkled structures, which reduces the achievable surface area growth.

The phase of growth after each shock-flame interaction is followed by a second phase, where 
Vortex Dynamics and Fractal Structures in Reactive and Nonreactive RMI

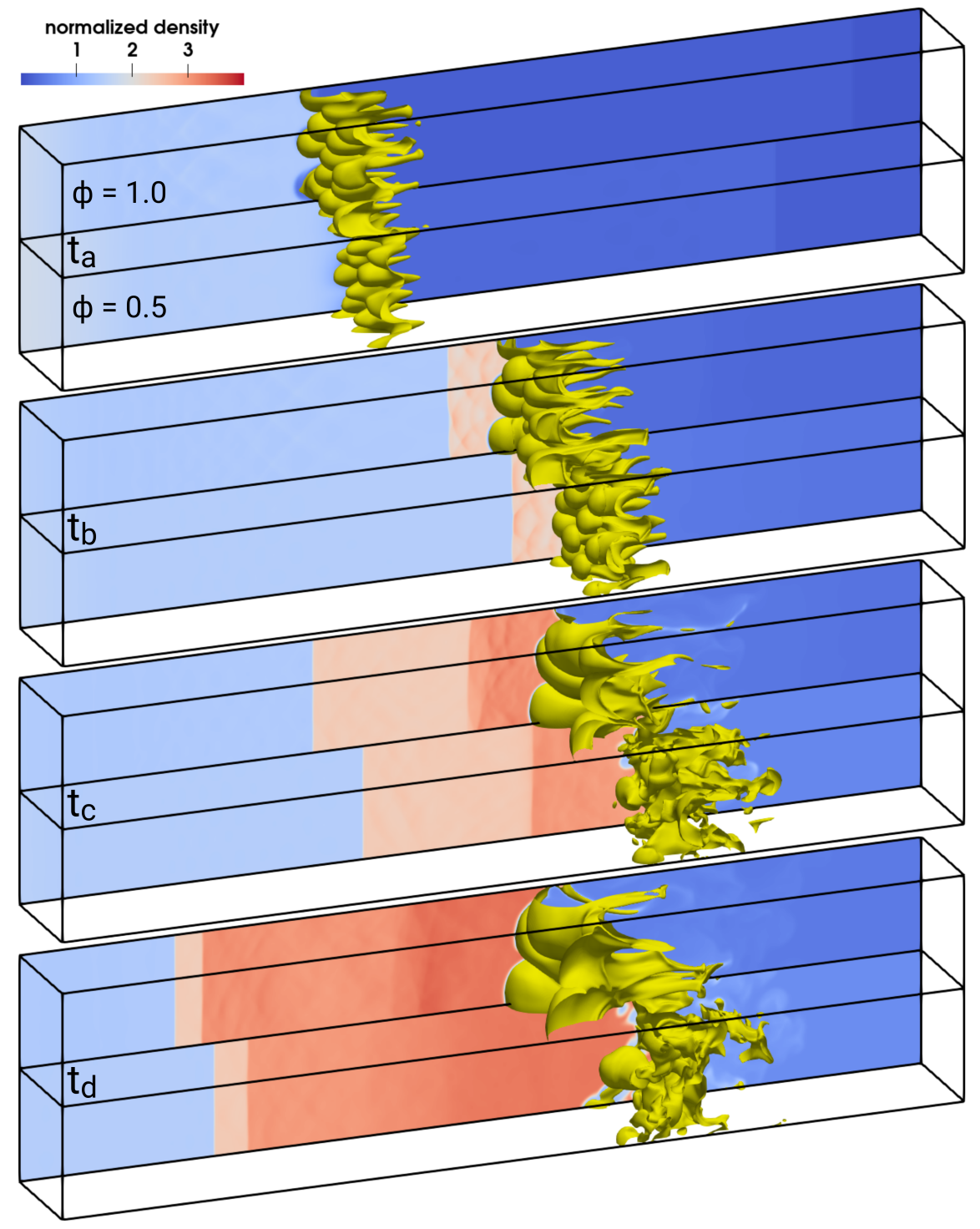

FIG. 8. 3D view and normalized density slice of the shock-flame interaction for $\phi=1.0$ (top half) and $\phi=0.5$ (bottom half). Timesteps $t_{\mathrm{a}}, t_{\mathrm{b}}, t_{\mathrm{c}}, t_{\mathrm{d}} \times S_{\mathrm{L}, \mathrm{st}} / \delta_{\mathrm{th}, \mathrm{st}}=0.2,0.4,0.6,0.8$. The flame is represented by a yellow iso-surface at $c=0.5$.

$A_{\mathrm{f}} / A_{\mathrm{f}, \mathrm{n}}$ reaches a peak value before decreasing again. This decrease can be caused by several factors, including molecular diffusion, structures merging and thereby removing surface area and the already mentioned reactive burnout of emerging wrinkled structures. The main influencing factor in this phase of $A_{\mathrm{f}} / A_{\mathrm{f}, \mathrm{n}}$ development is the reaction rate of the gas mixture, which is shown by comparing the results with the nonreactive case at $\phi=1.0$. Deactivating the chemical reaction prevents the decrease of $A_{\mathrm{f}} / A_{\mathrm{f}, \mathrm{n}}$ after the first shock interaction and reduces it significantly for the reshock. The nonreactive case reaches similar values as the lean $\phi=0.5$ case, which indicates 
Vortex Dynamics and Fractal Structures in Reactive and Nonreactive RMI
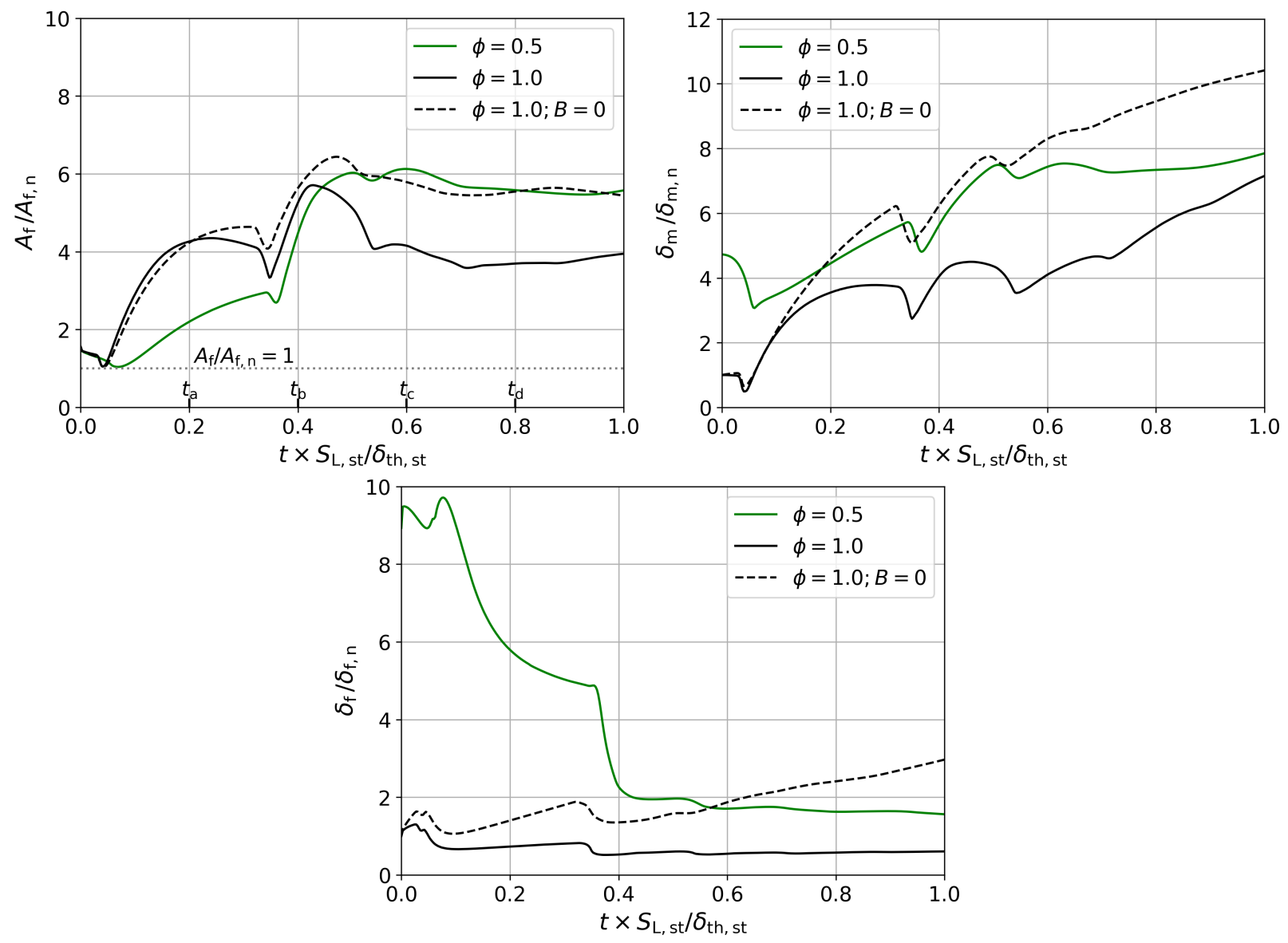

FIG. 9. Normalized flame surface area (top left), normalized mixing width (top right) and normalized flame thickness (bottom) over time for $\phi=0.5, \phi=1.0$ and nonreactive gas mixture.

that the latter case shows elements of passive scalar mixing (this will be further investigated in Sec. IV C). Another observation is the short drop to $A_{\mathrm{f}} / A_{\mathrm{f}, \mathrm{n}}=1$ during the first shock interaction, which is caused by the phase reversal effect explained in Fig. $1\left(A_{\mathrm{f}} / A_{\mathrm{f}, \mathrm{n}}=1\right.$ indicates a planar flame). The other drop, visible directly at the beginning of the reshock, is not related to the phase reversal effect, but is rather caused by the shock interacting with the leading flame structures first, before interacting with the rest of the flame. The overall rise in flame area due to the effects of the RMI is quite significant in both cases, with a maximum increase of around $400 \%$, compared to their measures at $t=0$.

Figure 9 (right) shows the development of the normalized mixing width $\delta_{\mathrm{m}} / \delta_{\mathrm{m}, \mathrm{n}}$ (Eq. (12c)) over time. In a nonreactive context, the mixing width represents the size of the mixing layer of heavy and light fluid, developing over time due to the RMI. While this explanation is also valid in the context of a reactive gas, there is an additional contribution to $\delta_{\mathrm{m}}$ from the flame thickness 
itself. This means that an unmixed (i.e. no (turbulent) mixing layer of unburned and burned gas) reactive gas will show a nonzero mixing width due to the thickness of the flame. This can be seen at $t=0$, where the initial value of $\delta_{\mathrm{m}} / \delta_{\mathrm{m}, \mathrm{n}}$ is about 4 times higher for $\phi=0.5$ than for $\phi=1.0$, due to the increased flame thickness of the lean case. While overall the $\delta_{\mathrm{m}} / \delta_{\mathrm{m}, \mathrm{n}}$ ratio is increasing over time due to the mixing effect of the RMI, the overlapping effect of flame thickness reduction due to the shock interaction causes short periods of decreasing mixing width. In the last phase of the simulation for $\phi=1.0$, large cusps of burned gas extend into the unburned mixture (Fig. 7, $t_{\mathrm{d}}$ ) causing the mixing width to grow. This effect is less pronounced for $\phi=0.5$ because of the lower reactivity, but balanced out by the higher flame thickness and increased wrinkling and mixing due to the RMI. The nonreactive case achieves the highest $\delta_{\mathrm{m}} / \delta_{\mathrm{m}, \mathrm{n}}$ ratio, which can be attributed to an increase in wrinkling and mixing in absence of reactive burnout. The absence of an reactive source term in the nonreactive case causes a steady increase of the interface thickness (nonreactive equivalent to the flame thickness) due to diffusion (Fig. 9). As indicated earlier this effect also contributes to the growth of the mixing width.

In order to further analyze the influence of $\phi$ on the development of the flame surface area and mixing width, a parametric analysis is performed (cases A, B, C and D in Tab. I). Starting with the case settings for $\phi=0.5$ the Lewis number (case A) and subsequently the reference Reynolds number (case B) are changed to the values used for $\phi=1.0$. For both cases this leads to a significant reduction of the flame thickness (Fig. 10), causing a higher density gradient across the flame and higher production of baroclinic torque. As seen in Fig. 10, this increases the $A_{\mathrm{f}} / A_{\mathrm{f}, \mathrm{n}}$ gradient at the first shock-flame interaction and higher peak values are reached than for $\phi=0.5$. For later times cases $\mathrm{A}$ and $\mathrm{B}$ decrease again to values close to $\phi=0.5$, due to surface reduction from diffusive effects. Case $\mathrm{C}$ additionally increases $T_{\mathrm{ad}}$ (higher $\tau_{\mathrm{h}}$ ), which again leads to a larger density gradient across the flame and to larger values of $A_{\mathrm{f}} / A_{\mathrm{f}, \mathrm{n}}$. Cases $\mathrm{A}, \mathrm{B}$ and $\mathrm{C}$ reduce the flame thickness and increase $T_{\mathrm{ad}}$ to the values for $\phi=1.0$ and both effects aid the development of RMI. The last performed variation $\mathrm{D}$ increases the reference Mach number $\mathrm{Ma}_{0}$, which can be interpreted as a dimensionless flame speed $\left(\mathrm{Ma}_{0}=S_{\mathrm{L}} / a_{0}\right)$ and therefore increases the reactivity to values very close to $\phi=1.0$. Now the already mentioned burnout effect becomes more significant and reduces the reachable $A_{\mathrm{f}} / A_{\mathrm{f}, \mathrm{n}}$ values to the values seen for $\phi=1.0$. No variation for the Prandtl number is performed as the numbers are already quite similar for both $\phi$.

Analyzing the mixing width in Fig. 10 leads to similar conclusions as already discussed for Fig. 9. Again, the mixing width values are not only influenced by mixing of heavy and light fluids 
Vortex Dynamics and Fractal Structures in Reactive and Nonreactive RMI
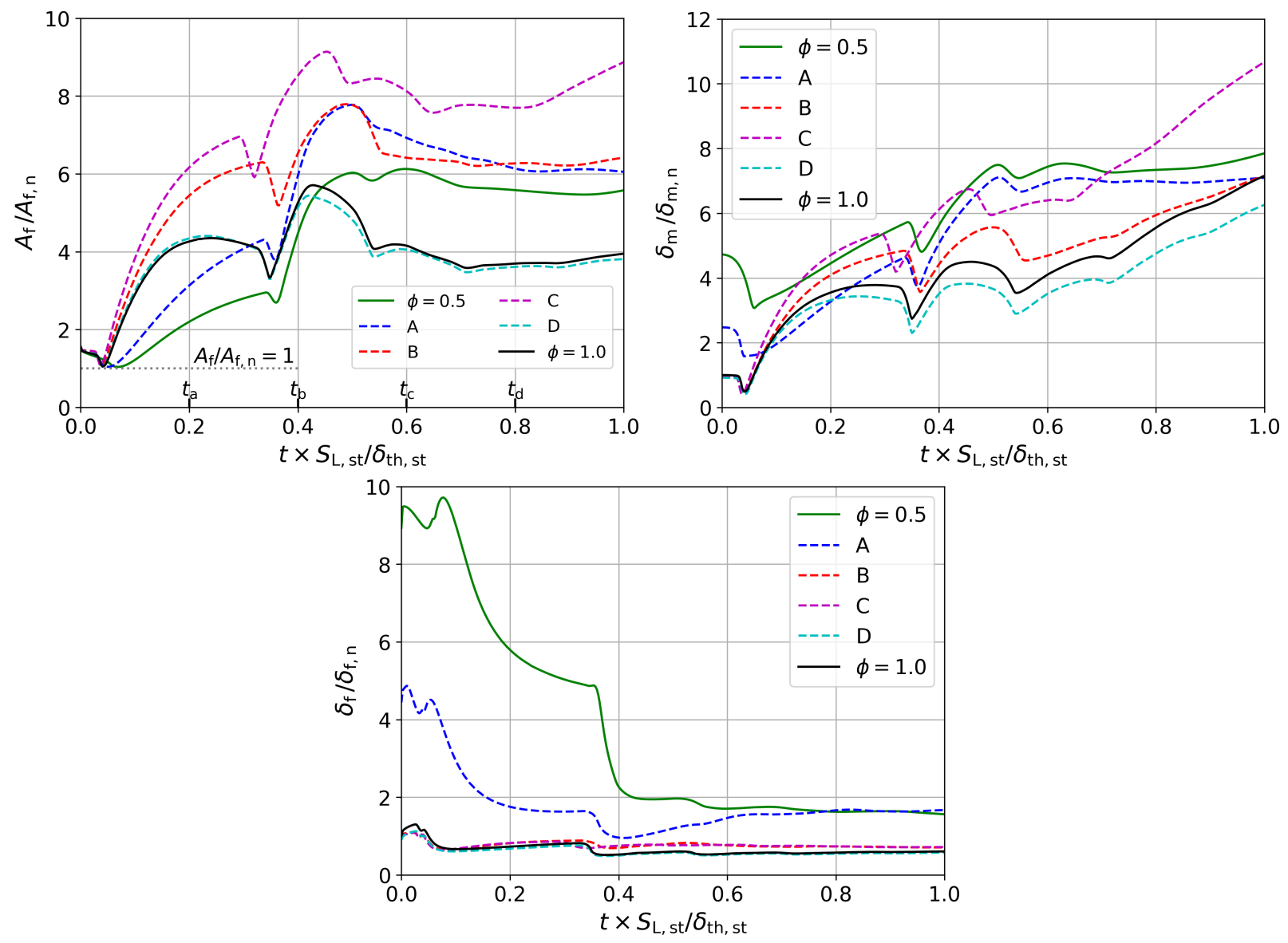

FIG. 10. Normalized flame surface area (left), normalized mixing width (right) and normalized flame thickness (bottom) over time for $\phi=0.5, \phi=1.0$ and cases A, B, C and D.

at the interface, but also by the changes in flame thickness. Although the interference of flame thickness and diffusion effects can make a detailed assessment difficult, the increased baroclinic torque (due to the higher density gradient across the flame) essentially leads to more mixing as measured by the increase of $\delta_{\mathrm{m}} / \delta_{\mathrm{m}, \mathrm{n}}$, while increasing the reactivity in case D reduces the mixing. Cases B, C and D show a similar behavior as $\phi=1.0$ towards the end of the simulation, where $\delta_{\mathrm{m}} / \delta_{\mathrm{m}, \mathrm{n}}$ keeps rising linearly.

Figure 11 shows a detailed view of the flame brush structure at time $t_{\mathrm{c}}$ and its transition from $\phi=0.5$ to $\phi=1.0$ via the cases A to D. Notable is the reduction in flame thickness from $\phi=$ 0.5 to case $\mathrm{B}$, leading to sharper defined structures. While the increased density gradient is less visible in case $C$, the change in reactivity from case $C$ to $D$ is clearly noticeable. The small wrinkled structures are burned out and only large bubble-like structures remain, which extend into the unburned mixture. 

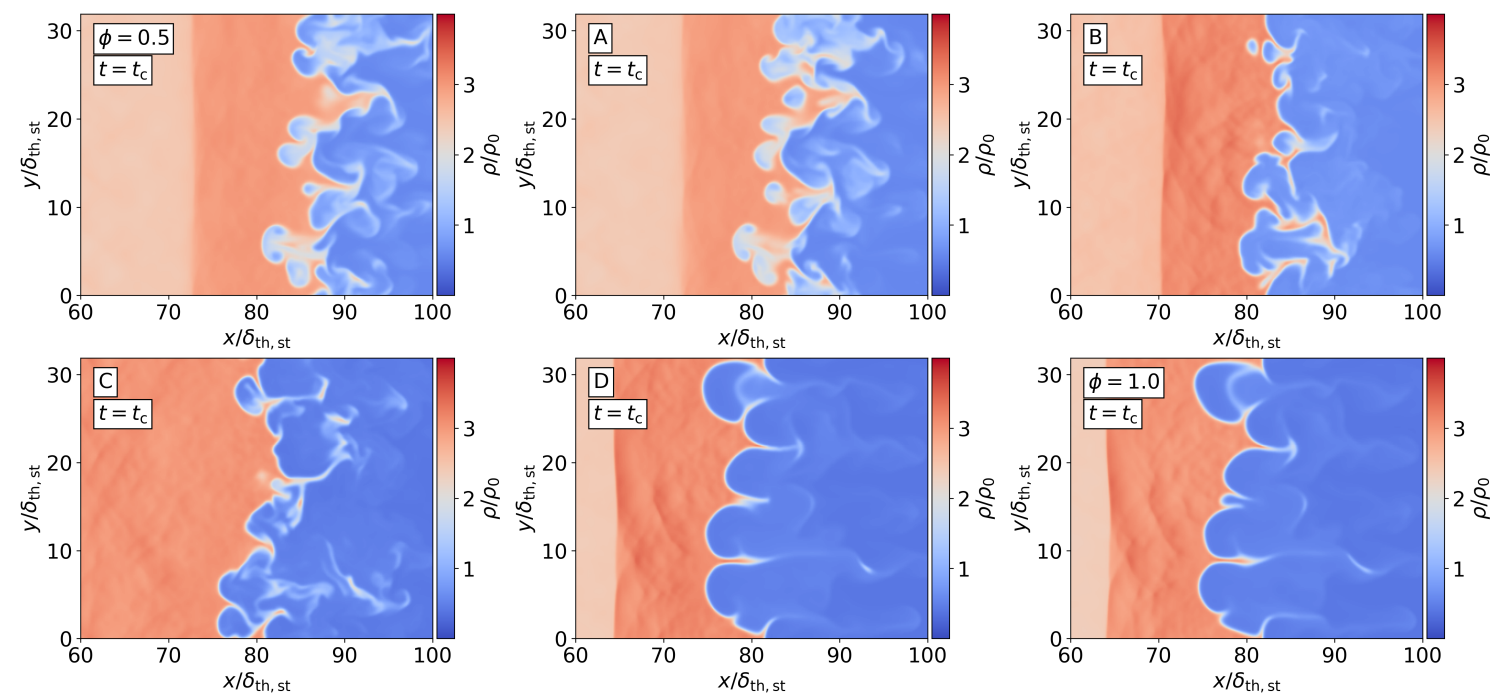

FIG. 11. Detailed view of density slice at $t=t_{\mathrm{c}}$ for $\phi=0.5, \phi=1.0$ and cases A, B, C and D.

Since the Lewis number is significantly smaller than unity for $\phi=0.5$, the flame might be more susceptible to thermo-diffusive instabilities in addition to the effects of the RMI. For the variation case A the Lewis number is increased to 1.1, while the other parameters correspond to the lean case. While this method does not allow to fully isolate the effects of RMI and thermo diffusive instabilities, it is supposed to give a general insight into how other instabilities overlay with the effects of the RMI. Increasing the Lewis number to 1.1 may decrease the susceptibility of the flame towards thermo diffusive instabilities, but as shown in Fig. 10 it will severely increase the effects of the RMI due to the decrease of the flame thickness.

A numerical parametric analysis conducted by Bambauer, Hasslberger, and Klein ${ }^{46}$ investigates the effect of $\mathrm{Ma}_{\mathrm{s}}$ and variations of the initial flame disturbance on the development of $A_{\mathrm{f}}$ and $\delta_{\mathrm{m}}$ for a nonreactive case $(\dot{\omega}=0)$. It is shown that increasing $\mathrm{Ma}_{\mathrm{s}}$ leads to higher values of $A_{\mathrm{f}}$ and $\delta_{\mathrm{m}}$, since the amount of baroclinic torque produced at the flame surface is directly dependent on the shock pressure gradient. The initial flame disturbance can also have a great impact on the development of the flame surface and mixing, since each maximum in the initial disturbance field (Fig. 4) acts as a source for the formation of fresh gas cusps. This can initially increase the flame surface area but also cause a loss, if the cusps interact and merge at later stages of the RMI. 
Vortex Dynamics and Fractal Structures in Reactive and Nonreactive RMI
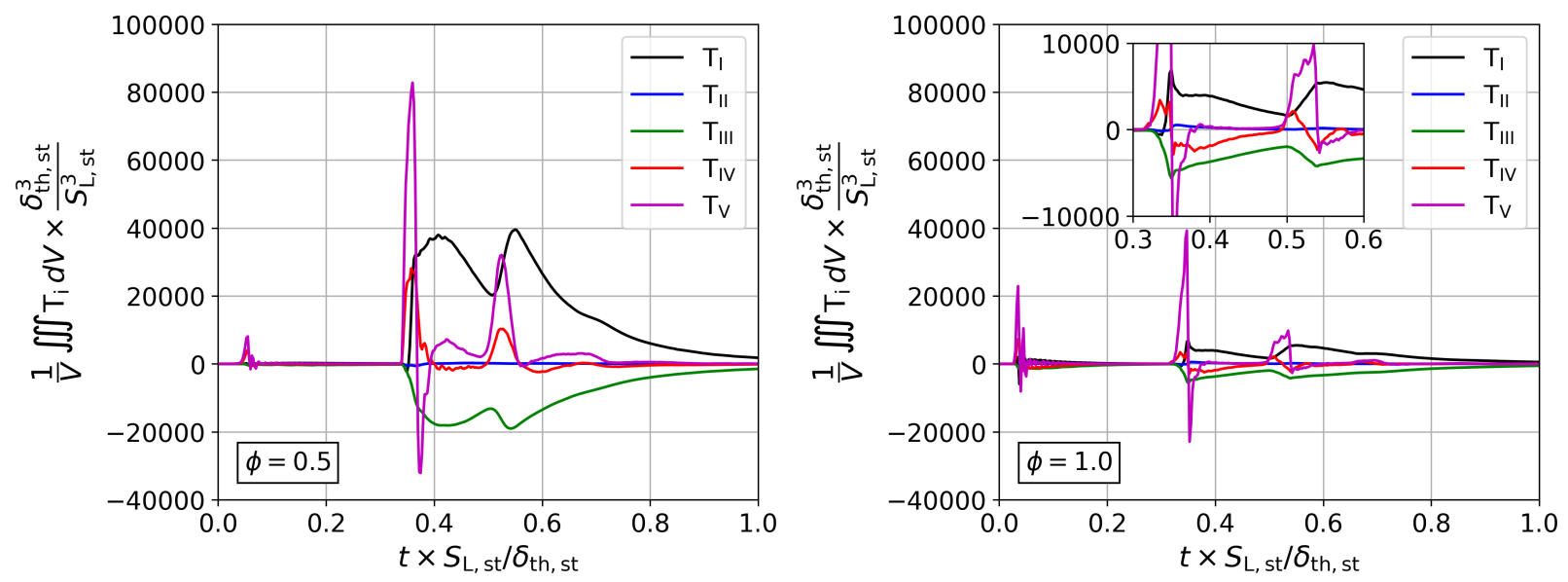

FIG. 12. Normalized volume integral (with $V=L_{\mathrm{x}} L_{\mathrm{y}} L_{\mathrm{z}}$ ) of the instantaneous enstrophy transport terms for $\phi=0.5$ (left) and $\phi=1.0$ (right).

\section{B. Enstrophy Analysis}

The generation of vorticity due to baroclinic torque at the flame surface, is the initiating mechanism for the RMI. The enstrophy $\Omega=\omega_{i}^{2} / 2$ can be interpreted as a scalar energy equivalent of the vorticity $\omega_{i}=\varepsilon_{i j k} \partial u_{k} / \partial x_{j}$ and its transport equation is given as:

$$
\frac{\partial \Omega}{\partial t}+u_{k} \frac{\partial \Omega}{\partial x_{k}}=\underbrace{\omega_{i} \omega_{k} \frac{\partial u_{i}}{\partial x_{k}}}_{\mathrm{T}_{\mathrm{I}}} \underbrace{-\varepsilon_{i j k} \omega_{i} \frac{1}{\rho^{2}} \frac{\partial \rho}{\partial x_{j}} \frac{\partial \tau_{k l}}{\partial x_{l}}}_{\mathrm{T}_{\mathrm{II}}}+\underbrace{\frac{\varepsilon_{i j k} \omega_{i}}{\rho} \frac{\partial^{2} \tau_{k l}}{\partial x_{j} \partial x_{l}}}_{\mathrm{T}_{\mathrm{III}}} \underbrace{-2 \frac{\partial u_{k}}{\partial x_{k}}}_{\mathrm{T}_{\mathrm{IV}}} \Omega+\underbrace{\varepsilon_{i j k} \frac{\omega_{i}}{\rho^{2}} \frac{\partial \rho}{\partial x_{j}} \frac{\partial p}{\partial x_{k}}}_{\mathrm{T}_{\mathrm{V}}}
$$

The terms on the right hand side represent the changes in enstrophy due to vortex stretching (I), viscous torque (II), dissipation (III), dilatation (IV) and baroclinic torque (V). A thorough discussion of the individual enstrophy transport terms in the context of turbulent combustion can be found in Lipatnikov et al. ${ }^{47}$ and Chakraborty et al. ${ }^{48}$. Figure 12 shows the volume integrals of the enstrophy transport terms for $\phi=0.5$ and $\phi=1.0$. Each peak in the baroclinic torque term (Term V) represents a shock-flame interaction. During the first and second shock-flame interaction the baroclinic torque dominates the overall enstrophy change, as indicated by the maximum reached peak height. At later times Terms I, III and IV significantly gain in importance and should be included in the discussion. At the first interaction the difference in flame thickness and adiabatic flame temperature leads to a higher peak in baroclinic torque for $\phi=1.0$ than for $\phi=0.5$. The situation is reversed at the reshock, where the decrease in flame thickness and build up of additional wrinkled structures on the flame surface support the production of baroclinic torque for $\phi=0.5$. 
Vortex Dynamics and Fractal Structures in Reactive and Nonreactive RMI
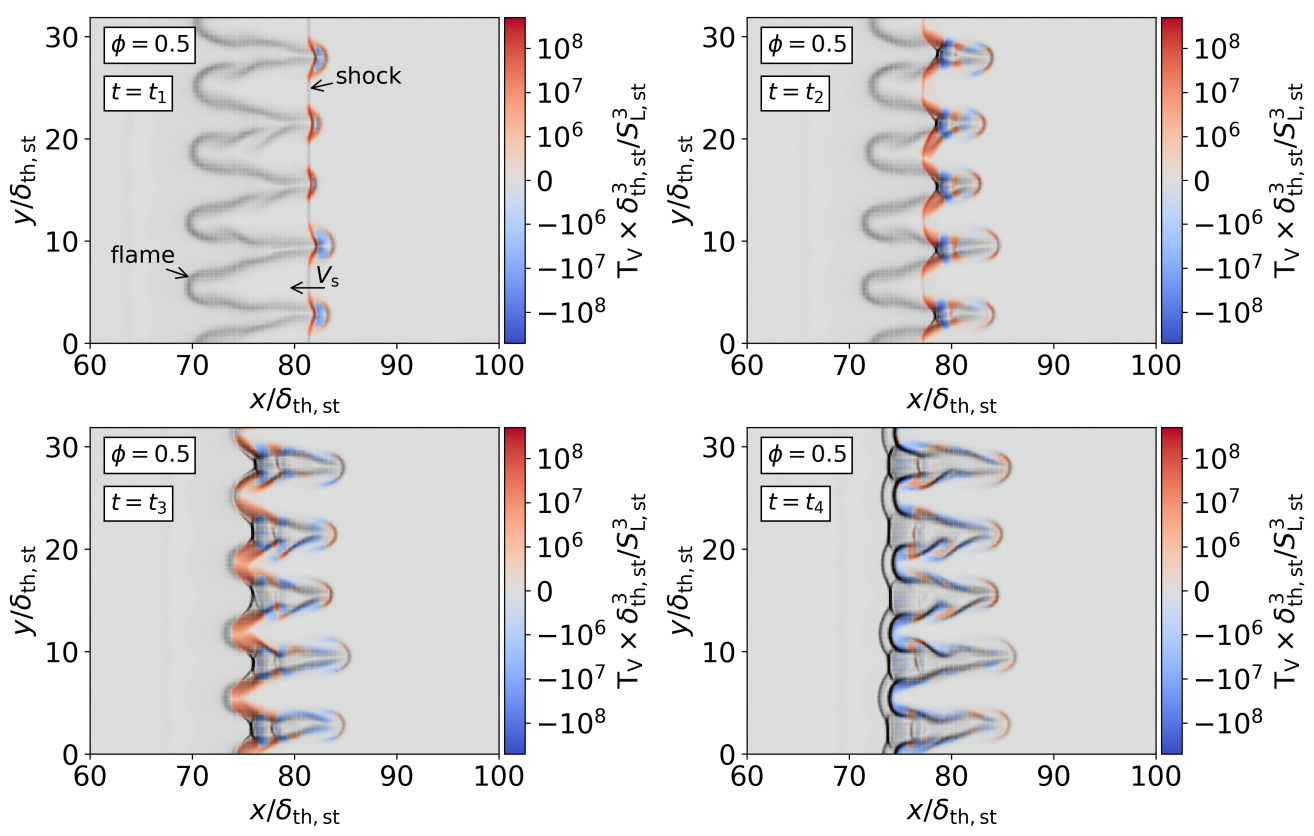

FIG. 13. Detailed view of baroclinic torque slice with numerical schlieren (in grayscale) at $t_{1}, t_{2}, t_{3}, t_{4} \times$ $S_{\mathrm{L}, \mathrm{st}} / \delta_{\mathrm{th}, \mathrm{st}}=0.345,0.355,0.365,0.375$ (during reshock) for $\phi=0.5$.

The third peak is caused by the interaction with the partially reflected shock wave shown in Fig. 7 at time $t_{\mathrm{c}}$. As shown in Fig. 13, local areas of positive and negative baroclinic torque contribution can be present during the entire period of the shock-flame interaction. Since Fig. 12 shows volume integrals, the negative and positive contributions partially cancel out each other, leaving only the net contribution for the entire domain. In case of the baroclinic torque the net contribution is positive at the beginning of the shock-flame interaction, meaning it acts as a source of enstrophy, but this is followed by a short period of negative net contribution, where it acts as a sink. A similar behavior is seen for the dilatation term (Term IV) at $\phi=1.0$, where the positive peak during the shock interaction is followed by a decrease to negative values at later times. Another interesting observation is the similarity of the vortex stretching (Term I) and dissipation (Term III) terms, which act in opposite directions effectively cancelling out each other (especially apparent for $\phi=1.0$ ). Figure 13 shows the planar shock wave evolving into a complex shaped shock pattern and multiple micro-reflections, as it is successively deflected and reflected when moving through the perturbed flame surface. After exiting the flame, the shock patterns merge into a planar shock again.

In order to asses the importance of the individual terms on the enstrophy development over time, the integral enstrophy budget is calculated for each term. This can be also interpreted as the 

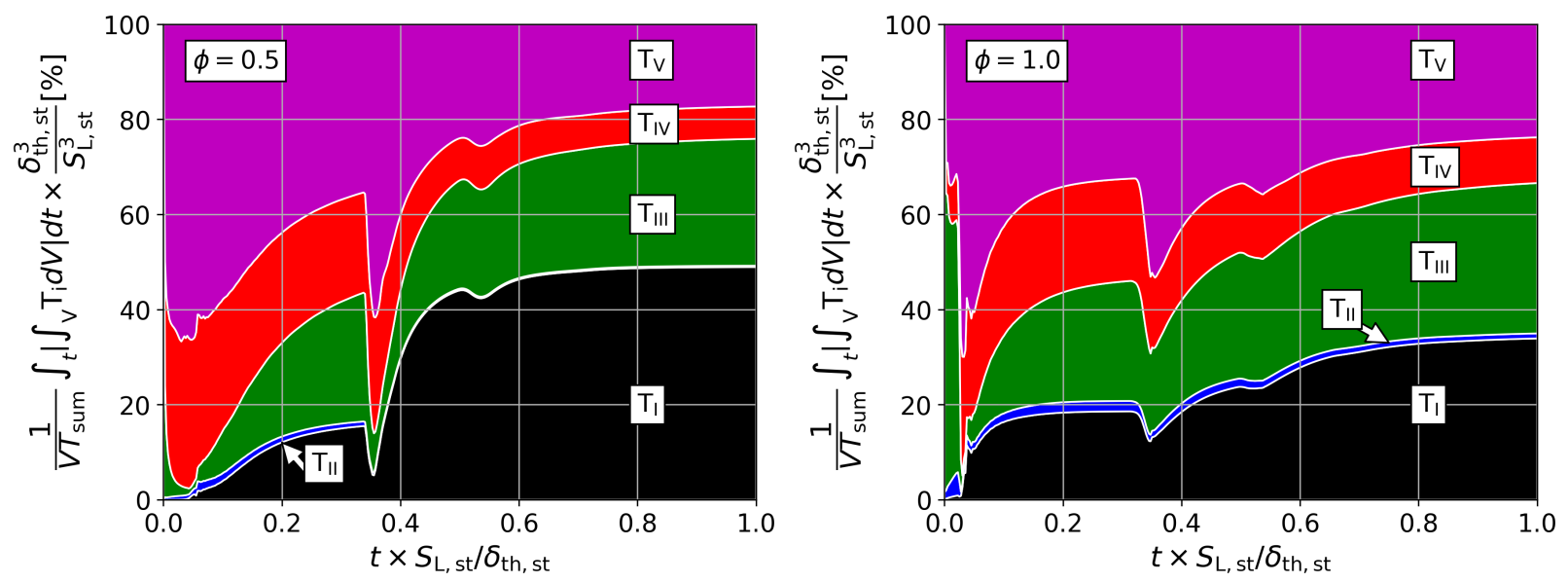

FIG. 14. Percentage share of the enstrophy transport terms on the sum of integral enstrophy budgets $T_{\text {sum }}$ over time for $\phi=0.5$ (left) and $\phi=1.0$ (right).

temporal development of the area integral below each graph in Fig. 12. The stacked plots in Fig. 14 show the percentage ratio of the integral enstrophy budget for each term to the total enstrophy budget $\mathrm{T}_{\text {sum }}$ over time. The moments of shock-flame interaction and therefore the spikes in baroclinic torque production are clearly visible in Fig. 14 for both cases, as Term V is responsible for 50-60\% of the total integral enstrophy budget in these moments. After the peak in baroclinic torque, the vortex stretching term (Term I) and dissipation term (Term III) become significantly more important. Towards the end of the simulation, Term I and Term III account for about $75 \%$ of the total integral enstrophy budget for $\phi=0.5$ and about $65 \%$ for $\phi=1.0$. As both terms act against each other (Fig. 12), Term III reduces Term I for $\phi=0.5$ and cancels out Term I for $\phi=1.0$. A similar result is obtained by $\mathrm{Cabot}^{49}$ for miscible 3D RT-simulations, where the baroclinic torque term is the dominant term in the initial stages of the RT instability but later the vortex stretching term becomes the dominant mechanism. For both cases the dilatation term (Term IV) takes a share of about 5-20\% throughout the whole simulation. While the influence of viscous torque (Term II) can be neglected in the present cases, an influence of about $10 \%$ is reported by Liu et al. ${ }^{50}$ for high shock Mach-numbers.

To gain a better insight of the influence of the equivalence ratio $\phi$ on the development of the enstrophy terms, the development of Term I and Term V (the two dominant terms) is compared for cases A, B, C and D. The effects of decreasing the flame thickness (mostly visible for case B) and increasing the adiabatic temperature (case C) all result in a significant increase of both terms. When the influence of an increased reaction rate is considered via case $\mathrm{D}$, the values of the terms 
Vortex Dynamics and Fractal Structures in Reactive and Nonreactive RMI
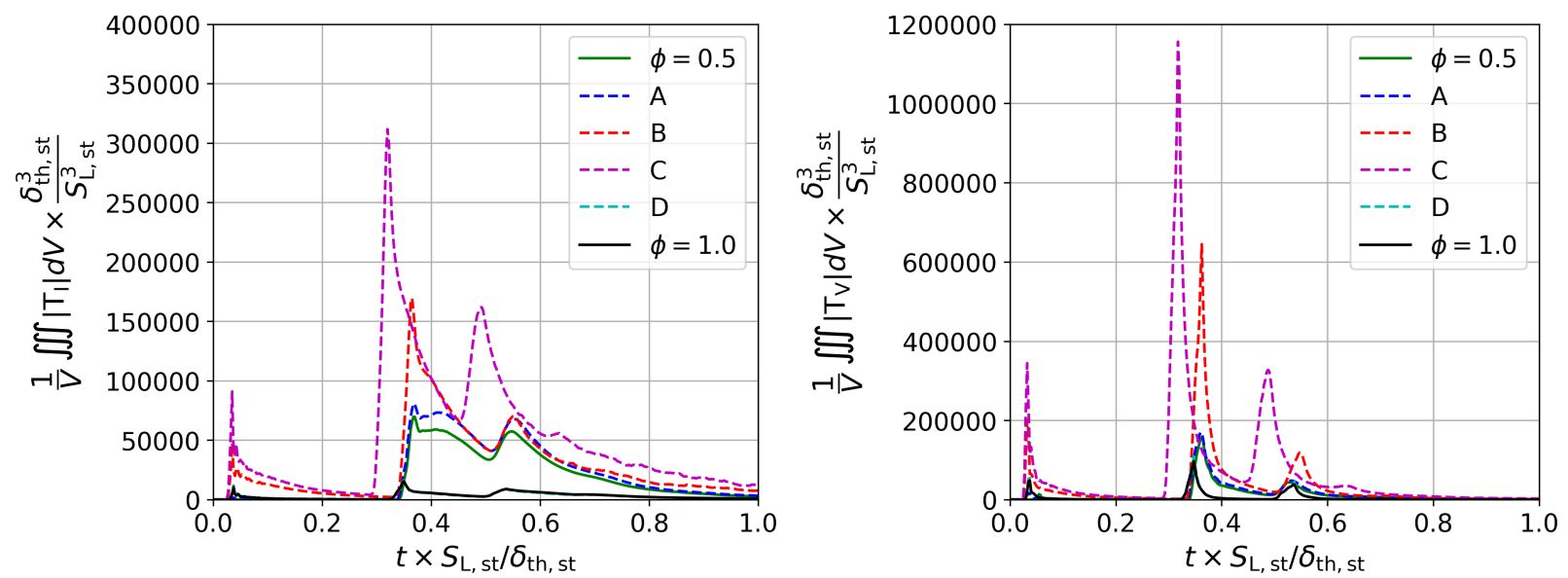

FIG. 15. Normalized volume integral (with absolute values) of the enstrophy transport terms I (left) and V (right) for $\phi=0.5, \phi=1.0$ and cases A, B, C and D. Note that the curves for D and $\phi=1.0$ collapse.

reduce significantly again to the values seen for $\phi=1.0$.

\section{Fractal Analysis}

A common approach for reaction rate closure in RANS and LES, is to model the sub-grid scale wrinkling factor $\Xi=A_{\mathrm{T}} / A_{\perp}=\overline{|\nabla c|} /|\nabla \bar{c}|$ (overbar indicates RANS averaging or LES filtering) as a power-law function, where $A_{\mathrm{T}}$ is the turbulent flame area and $A_{\perp}$ the projected flame area. If the filter width is chosen so that $A_{\perp}=A_{\mathrm{f}, \mathrm{n}}=L_{\mathrm{y}} L_{\mathrm{z}}$, then the normalized flame surface area in Fig. 9 can be also interpreted as a wrinkling factor $\Xi$. The power-law modeling results in the expression ${ }^{51,52}$ :

$$
\Xi=\left(\eta_{\mathrm{o}} / \eta_{\mathrm{i}}\right)^{D_{\mathrm{f}}-2}
$$

The outer and inner cut-off scales $\eta_{\mathrm{o}}$ and $\eta_{\mathrm{i}}$ are taken as the LES filter width $\Delta$ and a quantity that corresponds to the smallest occurring flame wrinkles ${ }^{53}$, respectively. For unity Lewis numbers the turbulent flame speed $S_{\mathrm{T}}=\int_{V}\left(\dot{\omega} / \rho_{\mathrm{ub}}\right) \mathrm{d} V / A_{\perp}$ (a fundamental quantity for reaction rate closure) can be related to the wrinkling factor $\Xi$ (strictly speaking for statistically planar flames) by invoking Damköhler's first hypothesis ${ }^{54}$ :

$$
S_{\mathrm{T}} / S_{\mathrm{L}}=\Xi=A_{\mathrm{T}} / A_{\perp}
$$

By explicitly filtering the present data, $\Xi$ and the projected flame area $A_{\perp}$ can be calculated ${ }^{55,56}$. The standard definition of $S_{\mathrm{T}}$ assumes a constant unburned gas density $\rho_{\mathrm{ub}}$. This assumption is 

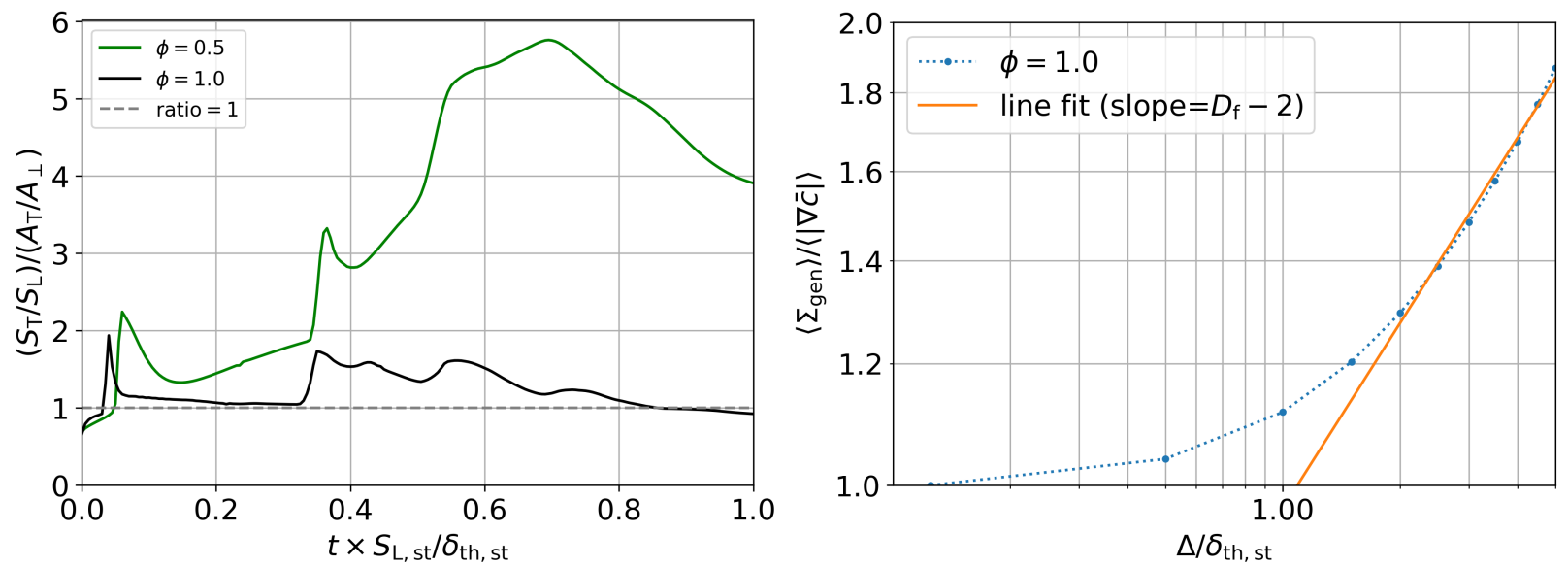

FIG. 16. Left: Deviation from the idealized Damköhler hypothesis for $\phi=0.5$ and $\phi=1.0$. Right: Double logarithmic plot of the volume averaged wrinkling factor over the normalized filter size for $\phi=1.0$ at $t \times S_{\mathrm{L}, \mathrm{st}} / \delta_{\mathrm{th}, \mathrm{st}}=1$

not valid in the present cases, since the the shock wave causes a density jump as it passes the flame. The corrected unburned density is calculated from the (partially) burned state of the gas mix for $\phi=1.0$ with $\rho_{\mathrm{ub}}=\rho\left(1+\tau_{\mathrm{h}} c\right)$, which is valid when the thermodynamic pressure remains unchanged. While this approach provides very good results for $\phi=1.0$, the approximation is not valid for Le $\ll 1(\phi=0.5)$. For $\phi=0.5$ the corrected unburned density can be approximated using the isentropic relation $\rho_{\mathrm{ub}}=\rho_{0}\left(p / p_{0}\right)^{1 / \gamma}$. In principle, the idea is to approximate a corrected unburned density from the (partially) burned state of the flame (using $\rho$ and $p$ ). For an unshocked burned state, the unshocked unburned density is calculated. For a shocked burned state, the shocked unburned density is calculated. The key to both approaches is that no explicit shock treatment is necessary, since the shock effects are already included in the values of $\rho$ and $p$. Deviations of the ratio $\left(S_{\mathrm{T}} / S_{\mathrm{L}}\right) / \Xi$ from unity specify the departure from Damköhler's hypothesis shown in Eq. (15). Figure 16 shows that for $\phi=1.0$ the Damköhler hypothesis is met very well, with minor deviations during shock-flame interactions and after reshock. In comparison, the deviations are significantly higher for $\phi=0.5$, since the Damköhler hypothesis is not valid for Le $\ll 1$ (see Chakraborty et al. $^{57}$ ).

The method of explicitly filtering the data also allows to calculate the fractal dimension $D_{\mathrm{f}}$ of the premixed flame. With the generalized flame surface density (FSD) $\Sigma_{\text {gen }}=\overline{|\nabla c|}$ defined by Boger et al. ${ }^{40}$ and using Eq. (14) the following expression for $\Sigma_{\text {gen }}$ is obtained ${ }^{58}$ : 


$$
\Sigma_{\text {gen }}=|\nabla \bar{c}|\left(\Delta / \eta_{\mathrm{i}}\right)^{D_{\mathrm{f}}-2}
$$

Following the method described in detail by Chakraborty and Klein ${ }^{55}$ and taking the volume average of Eq. (16), results in

$$
\log \left(\left\langle\Sigma_{\mathrm{gen}}\right\rangle /\langle|\nabla \bar{c}|\rangle\right)=\left(D_{\mathrm{f}}-2\right) \log (\Delta)-\left(D_{\mathrm{f}}-2\right) \log \left(\eta_{\mathrm{i}}\right)
$$

Equation (17) can be interpreted as a straight line equation with a slope of $\left(D_{\mathrm{f}}-2\right)$. Figure 16 (right) shows a double logarithmic plot of $\left\langle\Sigma_{\mathrm{gen}}\right\rangle /\langle|\nabla \bar{c}|\rangle$ over the normalized filter size $\Delta / \delta_{\mathrm{th}, \mathrm{st}}$ for $\phi=1.0$ and $t \times S_{\mathrm{L}, \mathrm{st}} / \delta_{\mathrm{th}, \mathrm{st}}=1$. When the filter width is smaller than the stoichiometric flame thickness $\delta_{\mathrm{th}, \mathrm{st}}$, the variation of $\log \left(\left\langle\Sigma_{\text {gen }}\right\rangle /\langle|\nabla \bar{c}|\rangle\right)$ becomes increasingly non-linear. For $\Delta \gg \delta_{\mathrm{th}, \mathrm{st}}$ the linear behavior expected from Eq. (17) becomes apparent and the fractal dimension $D_{\mathrm{f}}$ can be calculated from the slope of a line fit. By repeating this procedure for successive timesteps and for all cases, the temporal evolution of $D_{\mathrm{f}}$ (Fig. 17) can be determined. Since the definition of $D_{\mathrm{f}}$ is mathematically related to the FSD or flame surface area $A_{\mathrm{f}}$ (Eq. (12a)), it is consistent that $D_{\mathrm{f}}$ evolves similarly to $A_{\mathrm{f}}$ as shown in Fig. 9. The upper half of Fig. 17 includes a comparison to the nonreactive case. For the lower half of Fig. 17 additional parameter variations at equivalence ratios between $\phi=1.0$ and $\phi=0.5$ have been performed, for which the parameters can be found in Tab. I. At the start of the simulation, the shock flattens the initially disturbed (Fig. 4) flame surface, reducing the fractal dimension to $D_{\mathrm{f}} \approx 2$, or $\Xi \approx 1$. The fractal dimension then steeply increases to about 2.9 for $\phi=1$ and 2.8 for $\phi=0.5$. After the reshock $D_{\mathrm{f}}$ increases further for $\phi=0.5$ and reaches a maximum of $\approx 3(\approx 2.9$ for $\phi=1.0)$. The values obtained for $D_{\mathrm{f}}$ are limited to $D_{\mathrm{f}} \leq 3$, since the highest theoretically obtainable value for the fractal dimension in three dimensional space equals 3 (although higher values could be possible by interpreting Eq. (16) merely as a power law model). Especially in the middle sections of the simulation, values that reach that theoretical threshold should be interpreted with care, since they are subject to uncertainties due to sensitivities in the application of the post processing methods (filtering methods, straight line fit). The values for $D_{\mathrm{f}}$ reached towards the end are more conclusive as the values settle at about $2.4(\phi=1)$ and 2.7 $(\phi=0.5)$. The nonreactive case shows an interesting behaviour, as in this case the fractal dimension initially evolves in a similar fashion as for $\phi=1.0$ (the basis setup of the nonreactive case), but later settles at a similar value as the $\phi=0.5$ case. This further highlights the strong influence of the reactivity on the development of flame wrinkling.

The intersection of the straight line fit with the line given by $\left\langle\Sigma_{\text {gen }}\right\rangle /\langle|\nabla \bar{c}|\rangle=1$ in Fig. 16 yields 

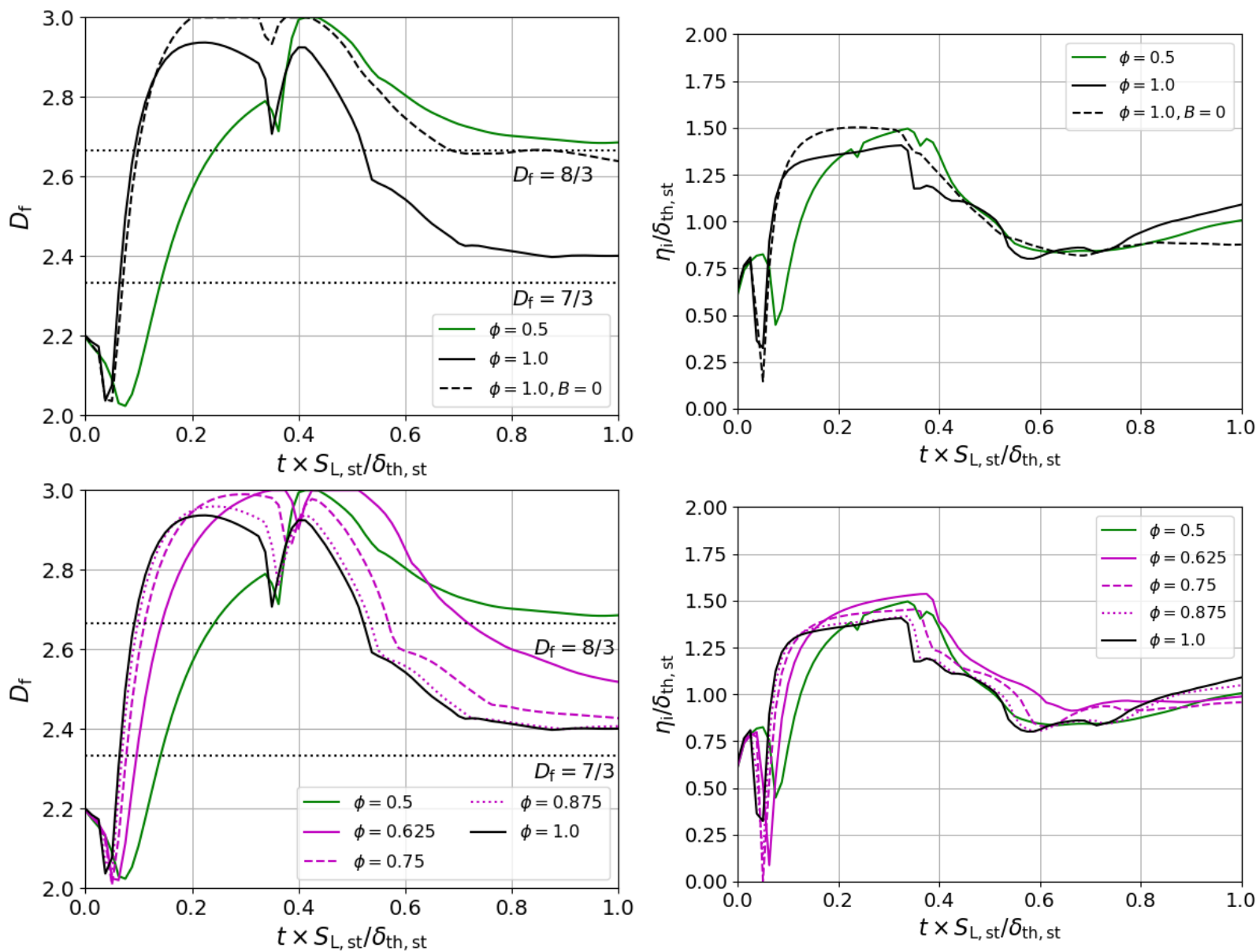

FIG. 17. Fractal dimension (left column) and inner cut-off scale (right column) for $\phi=0.5, \phi=1.0$ and nonreactive gas mixture (top) and a variation of equivalence ratios (bottom).

the inner cut-off scale $\eta_{\mathrm{i}}$. The development over time of the normalized inner cut-off scale $\eta_{\mathrm{i}} / \delta_{\mathrm{th}, \mathrm{st}}$ is shown in Fig. 17, where the stoichiometric thermal flame thickness $\delta_{\mathrm{th}, \mathrm{st}}$ is used for normalization in both cases. The values of $\eta_{\mathrm{i}} / \delta_{\mathrm{th}, \mathrm{st}}$ are only weakly dependent on the chosen equivalence ratio, since both cases show a similar temporal development for $\eta_{\mathrm{i}}$. The values start at $\approx 0.75 \delta_{\mathrm{th}, \mathrm{st}}$, then decrease to $\approx 0.5 \delta_{\mathrm{th}, \mathrm{st}}$, before sharply increasing to a maximum of $1.5 \delta_{\mathrm{th}, \mathrm{st}}$. Towards the end the inner cut-off scale stabilizes at around $1 \delta_{\mathrm{th}, \mathrm{st}} \pm 25 \%$. The assumption of a (nearly) constant and stoichiometry-independent $\eta_{\mathrm{i}}$ could be useful for simplified sub-grid modelling approaches using Eq. (16), as only $D_{\mathrm{f}}$ has to be modelled or specified depending on the case. An additional parameter variation of the equivalence ratio is shown in the bottom half of Fig. 17. Since the corresponding equivalence ratios are distributed uniformly $(\Delta \phi=0.125)$, the nonlinear nature of transition between the lean and stoichiometric reference cases becomes apparent. The growth rate of $D_{\mathrm{f}}$ after the first shock interaction is dependent on the equivalence ratio and decreases with 

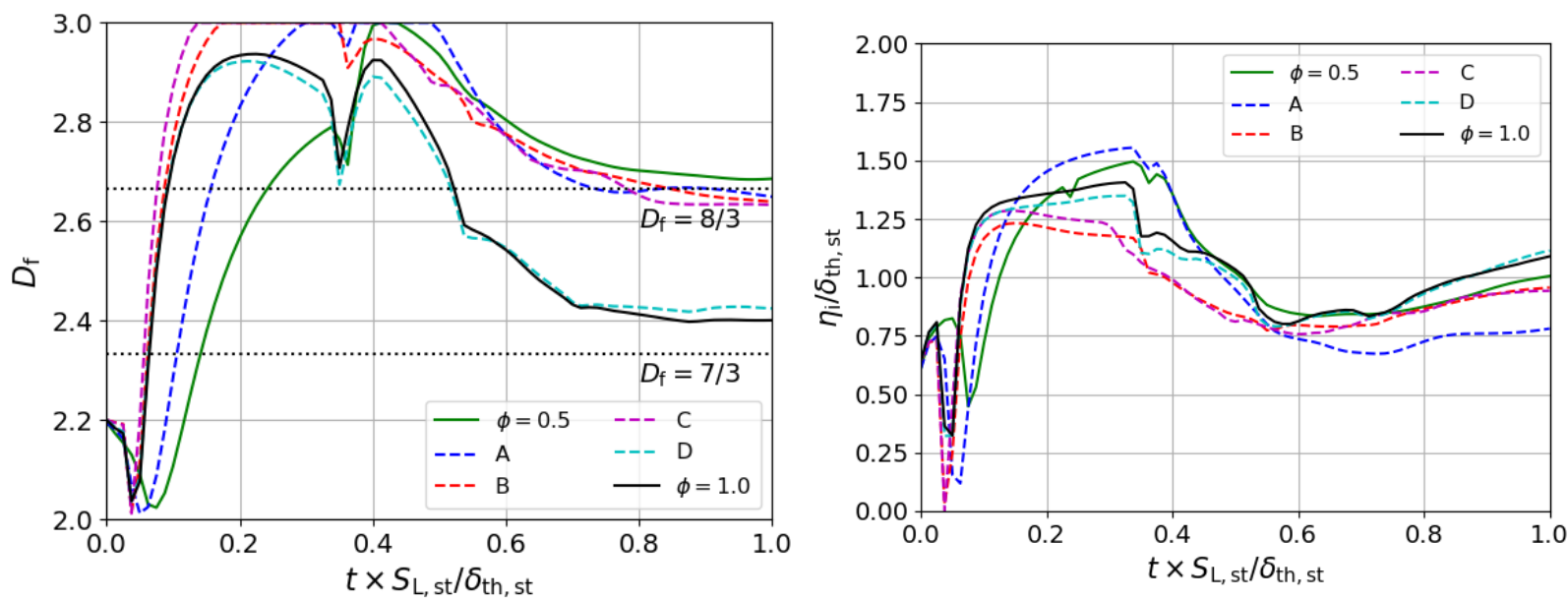

FIG. 18. Fractal dimension (left) and inner cut-off scale (right) for $\phi=0.5, \phi=1.0$ and cases A, B, C and D.

decreasing $\phi$. This behaviour is consistent with the development of the Mikaelian time scale ${ }^{30}$ $t_{\mathrm{m}} \sim \delta_{\mathrm{m}, 0} / \Delta v$, where $\delta_{\mathrm{m}, 0}$ is the initial mixing width and $\Delta v$ is the velocity jump at the mixing interface caused by the shock. Decreasing $\phi$ causes $\delta_{\mathrm{m}, 0}$ to increase, since this value is heavily dependent on the initial flame thickness. For the first shock-flame interaction $\Delta v$ equals the post shock velocity, which decreases with decreasing $\phi$, due to the dependence of the post shock velocity on the speed of sound $a_{0}=\sqrt{\gamma R_{\mathrm{S}} T_{0}}$, where $R_{\mathrm{S}}$ is mixture dependent. Both effects (increasing $\delta_{\mathrm{m}, 0}$; decreasing $\Delta v$ ) cause $t_{\mathrm{m}}$ to increase with decreasing $\phi$, explaining the longer growth period of $D_{\mathrm{f}}$ after the first shock-flame interaction. For lower values of $\phi$ there is less burnout of the emerging flame cusps, therefore higher values are reached for $D_{\mathrm{f}}$. For $\phi=0.5$ the re-shock interaction takes place while $D_{\mathrm{f}}$ is still in its growth phase, causing a lower peak to be reached after the first shock-flame interaction. Towards the end of the simulation the fractal dimension settles between values of $D_{\mathrm{f}}=7 / 3$ and $D_{\mathrm{f}}=8 / 3$, depending on the equivalence ratio. Here, a highly non-linear dependency of $D_{\mathrm{f}}$ on $\phi$ becomes apparent. The parameter variation confirms the previous observation, that the inner cut-off scale seems to be only weakly dependent on $\phi$ for the investigated cases.

The parametric analysis shown in Fig. 18 reaffirms the statements made in the previous sections towards the importance of the reactivity on the development of the RMI. While the previous variations $\mathrm{A}, \mathrm{B}$ and $\mathrm{C}$ have no noticeable impact on $D_{\mathrm{f}}$ towards the end of the simulation, changing the laminar flame speed $S_{\mathrm{L}}$ (and therefore the reactivity) to the values for $\phi=1.0$ leads to a sharp decline of $D_{\mathrm{f}}$ in case D. 
Vortex Dynamics and Fractal Structures in Reactive and Nonreactive RMI

The decrease in the equivalence ratio gives rise to reductions in $S_{\mathrm{L}}$ and increases in flame thickness, causing a shift from the strict flamelet regime towards a higher Karlovitz number regime. The end values reached for $D_{\mathrm{f}}$ (Fig. 17 and Fig. 18 ) increase from $\approx 7 / 3$ to $\approx 8 / 3$ when the equivalence ratio is being decreased. These values are consistent with findings in the literature as in the flamelet regime Kerstein ${ }^{59}$ suggests a fractal dimension of $7 / 3$, while the maximum $D_{\mathrm{f}}$ for a flame in the well-mixed regime ${ }^{60}$ as well as a passive scalar iso-surface ${ }^{61}$ has been found to be $8 / 3$.

\section{SUMMARY AND CONCLUSION}

In this study, the development of the RMI in lean $(\phi=0.5)$ and stoichiometric $(\phi=1.0)$ homogeneous $\mathrm{H}_{2}$ /Air mixtures was investigated by performing compressible 3D simulations of shockflame interactions with simple chemistry. As expected from theory, the interactions caused an increase in wrinkling and mixing as measured by the development of the flame surface area $A_{\mathrm{f}}$ and mixing width $\delta_{\mathrm{m}}$. The evolution of $A_{\mathrm{f}}$ after a shock interaction was divided into two separate phases. The first phase was characterized by an increase of $A_{\mathrm{f}}$, caused by the build up of fresh gas cusps (first shock interaction) and the development of wrinkled structures (mainly after reshock). The second phase was heavily influenced by the reactivity and characterized by a decrease of $A_{\mathrm{f}}$, due to transversal burnout of the fresh gas cusps and wrinkled structures. It was found that the equivalence ratio $\phi$ is an important factor in the development of the RMI, as it affects the reactivity, flame density gradient and speed of sound. The development of the mixing width $\delta_{\mathrm{m}}$ was found to be influenced not only by the mixing (from wrinkling) of unburned and burned fluid, but also by changes in the flame thickness itself. Similar to the behavior observed for $A_{\mathrm{f}}$, the shock interactions initially caused an increase in $\delta_{\mathrm{m}}$, while the burnout reduced the mixing. At later times, it was found that increasing the equivalence ratio (up to stoichiometric conditions) can also increase $\delta_{\mathrm{m}}$, as large flame cusps propagate into the unburned gas mixture.

An investigation of the enstrophy transport terms was performed, where the baroclinic torque was identified as the most dominant contributor, accounting for up to $60 \%$ of the total integral enstrophy budget at moments of shock-flame interaction. For the investigated cases it was found that, following the shock interactions, the contributions of the vortex stretching, dissipation and dilatation can become quite significant, accounting for up to $80 \%$ of the total integral enstrophy budget. This effect was found to be especially pronounced in the lean case, where vortex stretching alone accounted for up to $50 \%$ of the total integral enstrophy budget. The dissipation reduces the 
effect of the vortex stretching in the lean case and effectively cancels it out in the stoichiometric case. Finally, an investigation of the fractal behavior of the flame surface was conducted in the context of power-law based wrinkling factor modeling. Here, a highly non linear dependency of the fractal dimension $D_{\mathrm{f}}$ on $\phi$ was found, where decreasing $\phi$ causes an increase of $D_{\mathrm{f}}$ at late times. The inner cut-off scale however was found to be only weakly dependent on $\phi$, reaching values close to the thermal flame thickness of the stoichiometric flame $( \pm 25 \%)$.

In the future, it will be worthwhile to extend this analysis towards fuel-rich mixtures, thus decreasing laminar burning velocity (compared to stoichiometric conditions), whereas other important influencing parameters like Lewis number and speed of sound are further increasing.

\section{ACKNOWLEDGMENTS}

The presented work is funded by the German Federal Ministry of Economic Affairs and Energy (BMWi) on the basis of a decision by the German Bundestag (project no. 1501574) which is gratefully acknowledged. NC acknowledges financial and computational support from Engineering and Physical Sciences Research Council, UK (EP/R029369/1).

\section{DATA AVAILABILITY STATEMENT}

The data that support the findings of this study are available from the corresponding author upon reasonable request.

\section{REFERENCES}

${ }^{1}$ R. D. Richtmyer, “Taylor Instability in Shock Acceleration of Compressible Fluids," Communications on Pure and Applied Mathematics 13, 297-319 (1960).

${ }^{2}$ E. E. Meshkov, "Instability of the Interface of Two Gases Accelerated by a Shock Wave," Fluid Dynamics 4, 101-104 (1972).

${ }^{3}$ Rayleigh, "Investigation of the Character of the Equilibrium of an Incompressible Heavy Fluid of Variable Density," Proceedings of the London Mathematical Society s1-14, 170-177 (1882).

${ }^{4}$ G. Taylor, “The Instability of Liquid Surfaces when Accelerated in a Direction Perpendicular to their Planes. I," Proceedings of the Royal Society of London. Series A. Mathematical and Physical Sciences 201, 192-196 (1950). 
Vortex Dynamics and Fractal Structures in Reactive and Nonreactive RMI

${ }^{5}$ P. Ramaprabhu, V. Karkhanis, R. Banerjee, H. Varshochi, M. Khan, and A. G. Lawrie, "Evolution of the Single-Mode Rayleigh-Taylor Instability under the Influence of Time-Dependent Accelerations," Physical Review E 93, 1-17 (2016).

${ }^{6}$ Y. Zhou, "Rayleigh-Taylor and Richtmyer-Meshkov Instability Induced Flow, Turbulence, and Mixing. I,’ Physics Reports 720-722, 1-136 (2017).

${ }^{7}$ Y. Zhou, "Rayleigh-Taylor and Richtmyer-Meshkov Instability Induced Flow, Turbulence, and Mixing. II," Physics Reports 723-725, 1-160 (2017).

${ }^{8}$ Y. Zhou, R. J. Williams, P. Ramaprabhu, M. Groom, B. Thornber, A. Hillier, W. Mostert, B. Rollin, S. Balachandar, P. D. Powell, A. Mahalov, and N. Attal, "Rayleigh-Taylor and Richtmyer-Meshkov Instabilities: A Journey Through Scales,” Physica D: Nonlinear Phenomena , 132838 (2021).

${ }^{9}$ J. Lindl, "Development of the Indirect-Drive Approach to Inertial Confinement Fusion and the Target Physics Basis for Ignition and Gain,” Physics of Plasmas 2, 3933-4024 (1995).

${ }^{10}$ B. A. Remington, R. P. Drake, H. Takabe, and D. Arnett, "A Review of Astrophysics Experiments on Intense Lasers," Physics of Plasmas 7, 1641-1652 (2000).

${ }^{11}$ A. M. Khokhlov, E. S. Oran, and G. O. Thomas, "Numerical Simulation of Deflagration-toDetonation Transition: The Role of Shock-Flame Interactions in Turbulent Flames," Combustion and Flame 117, 323-339 (1999).

${ }^{12} \mathrm{G}$. Thomas, R. Bambrey, and C. Brown, "Experimental Observations of Flame Acceleration and Transition to Detonation Following Shock-Flame Interaction,” Combustion Theory and Modelling 5, 573-594 (2001).

${ }^{13}$ G. Ciccarelli, C. T. Johansen, and M. Parravani, "The Role of Shock-Flame Interactions on Flame Acceleration in an Obstacle Laden Channel," Combustion and Flame 157, 2125-2136 (2010).

${ }^{14}$ V. N. Gamezo, T. Ogawa, and E. S. Oran, "Flame Acceleration and DDT in Channels with Obstacles: Effect of Obstacle Spacing," Combustion and Flame 155, 302-315 (2008).

${ }^{15}$ S. B. Dorofeev, "Flame Acceleration and Explosion Safety Applications," Proceedings of the Combustion Institute 33, 2161-2175 (2011).

${ }^{16}$ W. Breitung, C. Chan, S. Dorofeev, A. Eder, B. Gelfand, M. Heitsch, R. Klein, A. Malliakos, J. Shepherd, E. Studer, and P. Thibault, "Flame Acceleration and Deflagration-to-Detonation Transition in Nuclear Safety," NEA/CSNI/R(2000)7 (2000). 
Vortex Dynamics and Fractal Structures in Reactive and Nonreactive RMI

${ }^{17}$ G. Ciccarelli and S. Dorofeev, "Flame acceleration and Transition to Detonation in Ducts," Progress in Energy and Combustion Science 34, 499-550 (2008).

${ }^{18}$ J. Hasslberger, Numerical Simulation of Deflagration-to-Detonation Transition on Industry Scale, Ph.D. thesis, Technical University Munich (2017).

${ }^{19}$ J. Yanez, M. Kuznetsov, and A. Souto-Iglesias, "An Analysis of the Hydrogen Explosion in the Fukushima-Daiichi Accident," International Journal of Hydrogen Energy 40, 8261-8280 (2015).

${ }^{20}$ K. W. Jenkins and R. S. Cant, "Direct Numerical Simulation of Turbulent Flame Kernels," in Recent Advances in DNS and LES, edited by D. Knight and L. Sakell (Springer Netherlands, Dordrecht, 1999) pp. 191-202.

${ }^{21}$ R. C. Rogers and W. Chinitz, "Using a Global Hydrogen-Air Combustion Model in Turbulent Reacting Flow Calculations," AIAA Journal 21, 586-592 (1983).

${ }^{22}$ T. Lu and C. K. Law, "Toward Accommodating Realistic Fuel Chemistry in Large-Scale Computations," Progress in Energy and Combustion Science 35, 192-215 (2009).

${ }^{23}$ C. Dopazo and E. E. O’Brien, "Functional Formulation of Nonisothermal Turbulent Reactive Flows," Physics of Fluids 17, 1968 (1974).

${ }^{24}$ S. Bane, J. Ziegler, and J. Shepherd, "Development of One-Step Chemistry Models for Flame and Ignition Simulation,” GALCIT Report GALTCITFM , 53 (2010).

${ }^{25}$ A. A. Wray, "Minimal Storage Time Advancement Schemes for Spectral Methods," NASA Ames Research Center, California, Report No. MS 202 (1990).

${ }^{26}$ G.-S. Jiang and C.-W. Shu, "Efficient Implementation of Weighted ENO Schemes,” Journal of Computational Physics 126, 202-228 (1996).

${ }^{27}$ C.-W. Shu, "Essentially Non-Oscillatory and Weighted Essentially Non-Oscillatory Schemes for Hyperbolic Conservation Laws," in Advanced Numerical Approximation of Nonlinear Hyperbolic Equations (Springer, 1998) pp. 325-432.

${ }^{28}$ T. Poinsot and D. Veynante, Theoretical and Numerical Combustion (Edwards, London, 2005).

${ }^{29}$ V. K. Tritschler, S. Hickel, X. Y. Hu, and N. A. Adams, "On the Kolmogorov Inertial Subrange Developing from Richtmyer-Meshkov Instability," Physics of Fluids 25, 071701 (2013).

${ }^{30}$ K. O. Mikaelian, "Extended Model for Richtmyer-Meshkov Mix,” Physica D: Nonlinear Phenomena 240, 935-942 (2011).

${ }^{31}$ J. D. Anderson, Fundamentals of Aerodynamics, McGraw-Hill series in aeronautical and aerospace engineering (McGraw Hill Education, 2017). 
Vortex Dynamics and Fractal Structures in Reactive and Nonreactive RMI

${ }^{32}$ G. Dimonte and M. Schneider, "Density Ratio Dependence of Rayleigh-Taylor Mixing for Sustained and Impulsive Acceleration Histories," Physics of Fluids 12, 304-321 (2000).

${ }^{33}$ M. M. Mansoor, S. M. Dalton, A. A. Martinez, T. Desjardins, J. J. Charonko, and K. P. Prestridge, "The Effect of Initial Conditions on Mixing Transition of the Richtmyer-Meshkov Instability," Journal of Fluid Mechanics 904, A3 (2020).

${ }^{34}$ B. Thornber, J. Griffond, O. Poujade, N. Attal, H. Varshochi, P. Bigdelou, P. Ramaprabhu, B. Olson, J. Greenough, Y. Zhou, O. Schilling, K. A. Garside, R. J. R. Williams, C. A. Batha, P. A. Kuchugov, M. E. Ladonkina, V. F. Tishkin, N. V. Zmitrenko, V. B. Rozanov, and D. L. Youngs, "Late-time Growth Rate, Mixing, and Anisotropy in the Multimode Narrowband Richtmyer-Meshkov Instability: The $\theta$-Group Collaboration,” Physics of Fluids 29, 105107 (2017).

${ }^{35}$ D. G. Goodwin, R. L. Speth, H. K. Moffat, and B. W. Weber, "Cantera: An object-oriented software toolkit for chemical kinetics, thermodynamics, and transport processes," https:// WWW . cantera. org (2018), version 2.4.0.

${ }^{36}$ J. K. Bechtold and M. Matalon, "The Dependence of the Markstein Length on Stoichiometry," Combustion and Flame 127, 1906-1913 (2001).

${ }^{37}$ E. E. O’Brien, “Turbulent Mixing of Two Rapidly Reacting Chemical Species,” Physics of Fluids 14, 1326 (1971).

${ }^{38}$ M. Klein, A. Herbert, H. Kosaka, B. Böhm, A. Dreizler, N. Chakraborty, V. Papapostolou, H. G. Im, and J. Hasslberger, "Evaluation of Flame Area Based on Detailed Chemistry DNS of Premixed Turbulent Hydrogen-Air Flames in Different Regimes of Combustion,” Flow, Turbulence and Combustion 104, 403-419 (2020).

${ }^{39}$ V. K. Tritschler, B. J. Olson, S. K. Lele, S. Hickel, X. Y. Hu, and N. A. Adams, "On the Richtmyer-Meshkov Instability Evolving from a Deterministic Multimode Planar Interface," Journal of Fluid Mechanics 755, 429-462 (2014).

${ }^{40}$ M. Boger, D. Veynante, H. Boughanem, and A. Trouvé, "Direct Numerical Simulation Analysis of Flame Surface Density Concept for Large Eddy Simulation of Turbulent Premixed Combustion,” Symposium (International) on Combustion 27, 917-925 (1998).

${ }^{41}$ D. Sharp, “An Overview of Rayleigh-Taylor Instability,” Physica D: Nonlinear Phenomena 12, 3-18 (1984).

${ }^{42}$ A. N. Lipatnikov, J. Chomiak, V. A. Sabelnikov, S. Nishiki, and T. Hasegawa, "Unburned Mixture Fingers in Premixed Turbulent Flames," Proceedings of the Combustion Institute 35, 1401-1408 (2015). 
Vortex Dynamics and Fractal Structures in Reactive and Nonreactive RMI

${ }^{43}$ A. Y. Poludnenko, "Pulsating Instability and Self-Acceleration of Fast Turbulent Flames," Physics of Fluids 27, 014106 (2015).

${ }^{44}$ N. Attal and P. Ramaprabhu, "Numerical Investigation of a Single-Mode Chemically Reacting Richtmyer-Meshkov Instability," Shock Waves 25, 307-328 (2015).

${ }^{45}$ R. Yu and A. N. Lipatnikov, "Direct Numerical Simulation Study of Statistically Stationary Propagation of a Reaction Wave in Homogeneous Turbulence,’ Physical Review E 95, 063101 (2017).

${ }^{46}$ M. Bambauer, J. Hasslberger, and M. Klein, "Direct Numerical Simulation of the Richtmyer-Meshkov Instability in Reactive and Nonreactive Flows," Combustion Science and Technology , 1-18 (2020).

${ }^{47}$ A. N. Lipatnikov, S. Nishiki, and T. Hasegawa, “A Direct Numerical Simulation Study of Vorticity Transformation in Weakly Turbulent Premixed Flames," Physics of Fluids 26, 105104 (2014).

${ }^{48}$ N. Chakraborty, I. Konstantinou, and A. Lipatnikov, "Effects of Lewis Number on Vorticity and Enstrophy Transport in Turbulent Premixed Flames," Physics of Fluids 28, 015109 (2016).

${ }^{49}$ W. Cabot, "Comparison of Two- and Three-Dimensional Simulations of Miscible RayleighTaylor Instability," Physics of Fluids 18, 045101 (2006).

${ }^{50}$ H. C. Liu, B. Yu, H. Chen, B. Zhang, H. Xu, and H. Liu, "Contribution of Viscosity to the Circulation Deposition in the Richtmyer-Meshkov Instability,’ Journal of Fluid Mechanics 895 (2020).

${ }^{51}$ C. Fureby, “A Fractal Flame-Wrinkling Large Eddy Simulation Model for Premixed Turbulent Combustion," Proceedings of the Combustion Institute 30, 593-601 (2005).

${ }^{52}$ R. Knikker, D. Veynante, and C. Meneveau, “A Dynamic Flame Surface Density Model for Large Eddy Simulation of Turbulent Premixed Combustion,” Physics of Fluids 16, L91-L94 (2004).

${ }^{53}$ O. Gülder, "Inner Cutoff Scale of Flame Surface Wrinkling in Turbulent Premixed Flames," Combustion and Flame 103, 107-114 (1995).

${ }^{54}$ G. Damköhler, "Der Einfluss der Turbulenz auf die Flammengeschwindigkeit in Gasgemischen," Zeitschrift für Elektrochemie und angewandte physikalische Chemie 46, 601-626 (1940).

${ }^{55}$ N. Chakraborty and M. Klein, "A Priori Direct Numerical Simulation Assessment of Algebraic Flame Surface Density Models for Turbulent Premixed Flames in the Context of Large Eddy Simulation," Physics of Fluids 20, 085108 (2008). 
Vortex Dynamics and Fractal Structures in Reactive and Nonreactive RMI

${ }^{56}$ N. Chakraborty, D. Alwazzan, M. Klein, and R. S. Cant, “On the Validity of Damköhler's First Hypothesis in Turbulent Bunsen Burner Flames: A Computational Analysis," Proceedings of the Combustion Institute 37, 2231-2239 (2019).

${ }^{57}$ N. Chakraborty, L. Wang, and M. Klein, "Streamline Segment Statistics of Premixed Flames with Nonunity Lewis Numbers," Physical Review. E, Statistical, Nonlinear, and Soft Matter Physics 89, 033015 (2014).

${ }^{58}$ F. Gouldin, K. Bray, and J.-Y. Chen, “Chemical Closure Model for Fractal Flamelets,” Combustion and Flame 77, 241-259 (1989).

${ }^{59}$ A. R. Kerstein, "Fractal Dimension of Turbulent Premixed Flames," Combustion Science and Technology 60, 441-445 (1988).

${ }^{60}$ E. R. Hawkes, O. Chatakonda, H. Kolla, A. R. Kerstein, and J. H. Chen, “A Petascale Direct Numerical Simulation Study of the Modelling of Flame Wrinkling for Large-Eddy Simulations in Intense Turbulence," Combustion and Flame 159, 2690-2703 (2012).

${ }^{61}$ B. B. Mandelbrot, "On the Geometry of Homogeneous Turbulence, with Stress on the Fractal Dimension of the Iso-Surfaces of Scalars,” Journal of Fluid Mechanics 72, 401 (1975). 